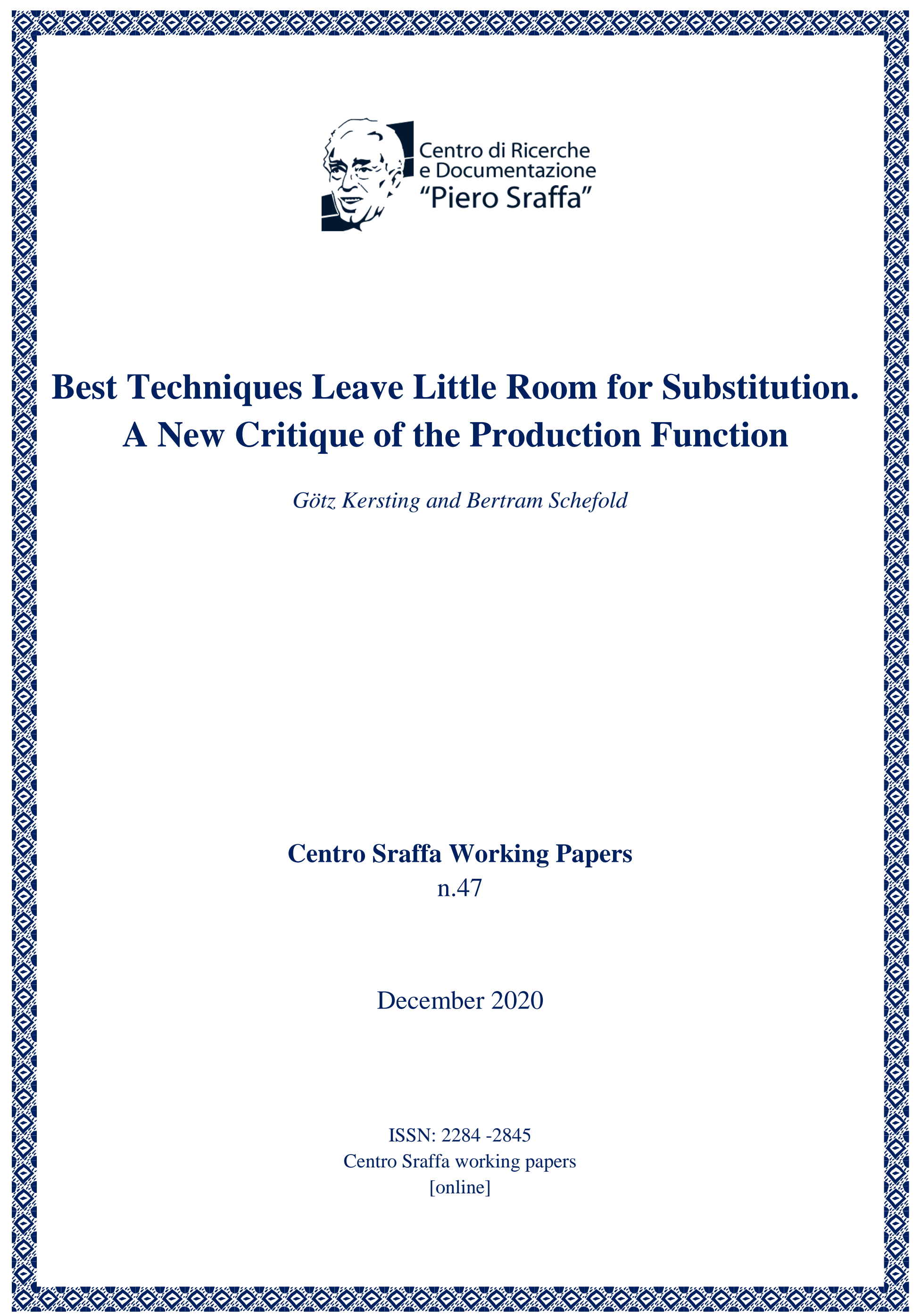




\title{
Best Techniques Leave Little Room for Substitution. A New Critique of the Production Function
}

\author{
Götz Kersting \\ Institut für Mathematik \\ Johann Wolfgang Goethe-Universität* \\ Bertram Schefold \\ Fachbereich Wirtschaftswissenschaften, RuW Fach 70 \\ Johann Wolfgang Goethe-Universität ${ }^{\dagger}$
}

December 15, 2020

\begin{abstract}
Samuelson assumed a linear wage curve for each of a continuum of techniques such that their envelope was a monotonically falling wage curve for the economy, from which an aggregate production function fulfilling the marginal productivity conditions could be derived. But the capital intensities of the techniques chosen at each rate of profit are not necessarily lower at higher rates of profit, if the wage curves are not linear, a possibility exemplified by reswitching. This critique of the capital controversy does not rule out Samuelson's construction as an approximation, since the paradoxes have been shown to be rare. Instead, a possibility is likely that has so far not been noticed: the envelope of the wage curves will in the relevant range of the rate of profit be dominated by a small number of efficient techniques of approximately equal capital intensity, leaving little room for substitution. A new mathematical theorem demonstrates that the expected number of techniques that appear on the envelope is given by $(2 / 3) \ln s$.
\end{abstract}

Key words: Capital theory, production function, substitution, reswitching B24, C62, C67, D57

\section{Introduction}

Paul A. Samuelson's article Parable and Realism in Capital Theory: The Surrogate Production Function, published 1962 in the Review of Economic Studies (Samuelson 1962), marked the beginning of what was probably the most conspicuous controversy in economic theory in the second half of the 20th century (Harcourt 1972; Kurz/Salvadori 1995). Only a few recall it today, although the discussion never ceased altogether (Kurz 2000; Hagemann 2020; Kurz 2020; Schefold 2020; Weizsäcker 2020). A brief summary of the debate seems indispensable before we come to what we regard as a very surprising new turnaround. It has been shown recently that the

*Robert-Mayer-Str. 10, 60325 Frankfurt am Main, kersting@math.uni-frankfurt.de

†Theodor-W.-Adorno-Platz 4, 60323 Frankfurt am Main, schefold@wiwi.uni-frankfurt.de 
pathologies of neoclassical capital theory, on which the early critiques were based, are quite rare in economies with many sectors (Schefold 2017), compared with the two or three sector models (Petri 2011; Mainwaring and Steedman 2000), which were predominantly used in the first phase of the debate. Even readers unfamiliar with it may remember that it had revealed the possibility of reverse capital deepening (Levhari 1965; Samuelson 1966). This means a change of technique, induced by a change of the rate of interest, such that an increase of this rate is associated with a higher intensity of capital and, similarly, a lowering of the real wage may induce the switch to a technique, which is less, not more labour-intensive. These anomalies can now be shown to be rare in large economic systems, both theoretically (Schefold 2016; Schefold 2018) and empirically (Han and Schefold 2006). If they were frequent, they would not only in theory, but also in applied economics question the working of the substitution mechanism on which the determination of equilibrium through supply and demand in capital and labour markets is based according to neoclassical theory. The new critique, to be presented below, concerns not only theory, but also economic policy. It predicts that the substitution possibilities are surprisingly few, even if the number of potential techniques in the given technology or "book of blueprints" is very large. It turns out that the intensity of capital is likely to stay virtually constant over the entire relevant range of variation of either the wage rate or the rate of profit. "Substitution possibilities" here refers to the efficient techniques that show up on the envelope of all wage curves, on the factor price frontier. A multiplicity of techniques and hence of substitution possibilities below the envelope remains, but they are inefficient. The new finding may thus help to explain the fact that major technologies stay in place, when the rates of wages and of interest change during the cycle, while minor activities may be affected: it may be easier to get personal for cleaning or for repairing cars, when unemployment rises and wages are depressed, but major technical changes follow from progress, not from shifts in distribution. The result is not due to rigidities or deviations from rational behaviour but follows from profit maximisation under conditions of perfect competition.

We shall first summarize the debate on capital theory; the notes summarize the essential mathematical background (Section 2 and Notes $1-7$ ). We shall then give an intuitive explanation of why there is little substitution and little change of the capital-labour ratio in the relevant range of the rate of profit (Section 3). Section 4 provides an exact mathematical statement of the main result, supported by experimental evidence. Section 5 discusses possible objections and suggests how the assumption of straight wage curves may be overcome in future research. Readers interested in the main mathematical result can go directly to Section 4, after having familiarized themselves with the problematic.

\section{The capital theory debate}

We start with a summary description of the origin of the debate. The marginal productivity theory of distribution had been controversial since its inception in the 1870s; Joan Robinson (1953-1954) and Piero Sraffa (1960) had criticized the concept of aggregate capital as its logical basis. Now Samuelson (1962) tried to defend it by deriving the aggregate production function from the set of possible steady states in a linear activities model.

To each level of distribution between labour and capital, characterized by the rate of interest, there corresponded a wage rate $w$, given the technique ${ }^{1}$. The factor price frontier $w(r)$ was monotonically falling and the same construct as the wage curve in Sraffa ${ }^{2}(1960)$. We here

refer to the Notes for formal details, which are well known for all connoisseurs of the critique so that references are not necessary. Samuelson succeeded in showing that a value of capital ${ }^{3}$, 
equal to the value of the capital goods used with the given technique at the steady state prices, could be calculated at each level of $r$ between zero and a maximum rate of profit or interest $R$. The efficient techniques ${ }^{4}$ (competitive and corresponding to the maximum attainable level of $w$ ) would change with the rate of profit such that the capital-intensity would increase, as the rate of interest was lowered (corresponding to the fundamental neoclassical proposition that lowering the rate of interest facilitates investment and accumulation), and such that lowering the wage rate would favour the adoption of labour-intensive techniques (corresponding to the fundamental neoclassical proposition that wages need to be lowered to increase employment). The envelope of the wage curve would express the same as a production function (see below Note 7).

Samuelson had assumed, as he himself had pointed out repeatedly, that the capital-intensities of the different sectors were the same in all industries of any given technique, so that the individual wage curves of each technique were straight lines ${ }^{5}$. But it was found in the subsequent controversy that the individual wage curves were not straight, their envelope, though falling, was therefore not necessarily convex; it could contain concave parts, hence the aggregate value of capital did not necessarily fall relative to labour employed, as the rate of interest or profit was increased, neither for the individual techniques, nor on the envelope. To the extent that this happened without a choice of technique and was due to the curvature of the individual wage curves on the envelope, these occurrences were called (non-neoclassical) Wicksell-effects. Even more striking was the phenomenon of reverse capital deepening. As the rate of interest increases, techniques change at the intersection of individual wage curves on the envelope. These are the so-called switchpoints on the envelope, where generically one method changes so that there are two systems of prices, differing in one method; prices are the same for both systems at the switch-point itself. It turned out to be possible that a technique, adopted because of a rise of the rate of interest across the switch-point, showed a higher, not a lower intensity of capital and indeed, a technique, which had been profitable at a low rate of profit and which was dominated by other methods at intermediate profit rates, could reappear at a high rate (so called reswitching) ${ }^{6}$. Samuelson's student Levhari (1965) tried to exclude reswitching by means of assuming that each technique was given by an indecomposable input-output matrix, but his proof was erroneous, counter-examples were provided by a number of critics, starting with Pasinetti (1966), and the story of this battle was told in in quite a few histories, short and long (Harcourt 1962; Kurz and Salvadori 1965; Hagemann 2020). After this, advanced theorists like Samuelson himself admitted that the use of the aggregate production function was not rigorous (Samuelson 1966), but it did not disappear from the textbooks and returned with the new theories of growth in the 1980s (Aghion and Howitt 1998).

Garegnani (1970), in what was perhaps the most thorough contribution to the critique, tried to show that Samuelson's assumption had, by being restrictive, not only been sufficient to show that the surrogate production function could be constructed, but Garegnani tried to demonstrate that these assumptions were also necessary, and not only for the validity of neoclassical theory in the form of the production function (the Clarkian parable) but also for Walras's general equilibrium (as a theory of long-run equilibrium) and for Böhm-Bawerk's analysis. As we discuss in the Notes $^{7}$ and partly in other papers (Schefold 2013a), Garegnani's proof of the necessity omitted a small possibility in the case of the aggregate production function and was more tentative than rigorous as regards the 19th century neoclassical economists. What was on that occasion in 1970 more important, he failed to clarify the relevance of his critique for modern general intertemporal equilibrium, which is normally not a long-run equilibrium because the initial endowments are not in general given in that proportion which would allow to reach a steady state from the beginning and could then be maintained for subsequent periods. The question as to how the critique related to intertemporal equilibrium had been raised by the very editor of the Journal 
(Bliss 1970). Garegnani endeavoured to answer to this challenge in later years, and this entailed a controversy of its own, which we cannot pursue here (Schefold 2008). Suffice it to say that non-neoclassical Wicksell-effects and reverse capital deepening imply a special kind of stability problems for general intertemporal equilibria, even if their existence is not in question.

By contrast, Garegnani's general assertion, that the critique affected all versions of the old long-run neoclassical theory, was rooted in his early work (Garegnani 1960) and turned out to be a profound insight. It has been developed later by Petri (2004), Schefold (2016), among others, and Garegnani's chief merit was perhaps his identification of the break in the history of neoclassical thought, when early long-run neoclassical theory ended, because Lindahl and Hicks opted successfully for the introduction of temporary equilibria (Garegnani 1976), with the result that the long-period method was largely abandoned by neoclassical theorists in favour of the intertemporal approach, except for the steady states, which were kept as a framework to discuss theories of growth (e. g. von Weizsäcker 1971).

But, in all this, nobody seemed to question or even to notice the least plausible of Samuelson's assumptions. What was noticed, and pointed out by Samuelson himself, was that the individual wage curves had to be straight lines, for then the capital-intensity was constant for each technique and hence an unambiguous characteristic of the wage curve: each technique had its specific capital-intensity. For the wage curves to be straight, the capital-intensities had to be the same in all sectors, as already stated, and this means that relative prices did not depend on distribution and therefore were equal to labour values. As Salvadori and Steedman (1988) have shown, methods of production taken from different techniques could thus not be combined, for the combinations would have led to unequal capital intensities in the different sectors, and the straight wage curves would at the intersections on the envelope have been dominated by the wage curves of combinations, which would thus in general not be straight ${ }^{8}$. Samuelson himself had very briefly indicated that the capital goods in his model were specific to the techniques. This means that each technique produces and uses capital goods that are not produced or used in other techniques, while we shall assume that methods taken from different techniques can be combined to form a new technique (but we shall discuss a restriction on technological choice as an intermediate case in Section 5). The critics have quite correctly pointed out the lack of generality of Samuelson's construction ${ }^{9}$, which is expressed in these properties, and they have noted the irony that Samuelson based the surrogate production function on the assumption that the labour theory of value held for each of the alternative techniques, as in the first two volumes of Das Kapital by Karl Marx, while he wanted to distance himself from the Marxian tradition (Samuelson 1966 [1961]).

But all the critics failed to perceive that Samuelson did not only assume that individual wage curves were straight, but also that wage curves of techniques with a high productivity of labour predominantly had a low maximum rate of profit or, with constant returns to scale, a low maximum rate of growth, equal to the maximum rate of profit. In his diagram, the techniques can be seen to be ordered according to the productivity of labour, equal to $w(0)$, the real wage per head, if all output goes to workers. Each technique is also characterized by a maximum rate of profit, and these two points determine the straight wage curve. Samuelson's assumption, as shown in his diagram, but not formulated in words, is that, as in Diagram 1, Section 3 below, the permutation of the maximum rates of profit associated with the technique is the inverse ordering of the ordering of the techniques according to the productivity of labour - except perhaps for a few inferior techniques, which do not get up on the envelope. If it is not assumed that the ordering of the maximum rates of profit is inverse to that of the productivities of labour or if not some similar assumption is made, not a few but most techniques will have wage curves below the envelope and the number of the techniques on the envelope will be so small that the idea of substitution (which is what all the discussion is about) looses its meaning. This is what we shall show. 


\section{From reswitching to the number of substitution possibil- ities}

How many possibilities of substitution do we encounter on the envelope? In order to clarify the question, it is useful to ask why a technique should not be 'good' both with regard to the productivity of labour and the maximum rate of growth. This question presumably never came up in the discussion, because neoclassical economists repressed the entire discussion in their minds and regarded the idea of substitution as obvious, while the critics thought that the wage curves of individual techniques were curves anyway, which formed a complicated pattern such that most of them came up on the envelope, on the factor price frontier, in unpredictable sequences. I (here Bertram Schefold is writing) can claim an exception for myself. It is now (spring 2020) exactly 50 years ago that I asked Joan Robinson in her class in Cambridge, whether she expected 'many' wage curves on the envelope. Sraffa himself had spoken of a "rapid succession of switches" (Sraffa 1960 , p. 85), which one would encounter as one moved down the envelope. She replied that she thought there would be only one wage curve dominating the others, that of the 'best' technique; it would be superior independently of the level of distribution. I was astonished. We often discussed the choice of techniques and switch-points, when we experimented with two-sector models (the calculations of prices derived from input-output models came later). When I asked her about visible changes of technique, she replied that that was technical progress. I now had a doubt in my mind, but I returned to my agenda, which was the theory of joint production.

In the early 2000s, a Korean student of mine, Zonghie Han, wanted to find empirical proofs for the existence of reswitching and reverse capital deepening. He examined the envelopes of wage curves, calculating the individual wage curves from empirical input-output tables, which, taking in combination as books of blueprints, gave rise to a rich choice of techniques. In order to keep the calculations manageable, he would always combine the input-output tables of two countries at one time or of one country at two different times, assuming that in each industry there was the choice between two methods, say, either car manufacturing French style or German style. Since the input-output tables had 33 relevant sectors, there were now $2^{33}$ potential techniques for the two countries taken together. If all the methods of production had been assumed to be specific for the technique in which they were used, and that means, in this example, for the country concerned, only two wage curves, one of France and one of Germany, would have been to be compared. Intermediate cases of specificity are plausible: Both Austria and Greece produce wine, but only Greece has maritime transportation. Since Han ruled out such specificity completely (which was of course as daring an assumption as the opposite $)^{10}$, two input-output tables gave rise to the full potential of a spectrum of $2^{33}$ different wage curves. The envelope of each spectrum was calculated by linear programming, and pairing a number of input-output tables in this manner, several hundred envelopes were obtained, which Han inspected painstakingly. A thesis resulted (Han 2003) and a joint paper (Han and Schefold 2006) containing two surprises: (1) Cases of reverse capital deepening and one case of reswitching could be found, but they were rare. More than $95 \%$ of the switches were neoclassical. (2) About ten wage curves of individual techniques formed each envelope; not one, as Joan Robinson had anticipated, and not many, as Samuelson and Sraffa had implied, without making their assumptions explicit - indeed, they did not even realize that they here were assuming something.

I (BS) have spent much time during the last fifteen years trying to confirm, reject or at least to understand these results. Before we get to puzzle (2), which is the subject of this paper, pointedly reformulated, we must summarise some results regarding (1). 
Numerous studies show that wage curves derived from input-output tables are never straight, but they are not as strongly curved as the critics used to expect. The reasons are not entirely clear. It is well known that a linear wage curve results if prices are expressed in terms of baskets of goods as numéraires and if the basket is proportional to Sraffa's standard commodity ${ }^{11}$. Appropriate measures for prices in such empirical comparisons are not individual goods - they, taken as numéraires, lead to more pronounced curvatures in most cases - but an average of the vectors of consumption or of the vectors of net national products in the comparison of the wage curves derived from the input-output systems of different countries in a given period. These vectors will in general not be equal to the standard commodity of any of the countries concerned, but their compositions may be somewhat similar, so that the deviation of the wage curves from linearity may be moderate ${ }^{12}$.

It is also known that linear wage curves result, if prices are equal to labour values (this, strictly assumed, was Samuelson's case). Actual prices deviate from labour values, but perhaps not that much, as already Ricardo thought ${ }^{13}$. Hence another reason why the deviation of actual wage curves from linearity may be moderate, and the two reasons given do not exclude, but may reinforce each other.

A third possibility was investigated on purpose in order to explain the phenomenon of near linearity of wage curves in the context of the critique of the surrogate production function. If the systems are random in that the input-output matrices are random (with certain additional properties) and if the labour vector stands in a certain random relationship to the matrix, a nearly linear wage curve will result, and it tends to strict linearity as the dimension of the system (the number of sectors) tends to infinity. These assumptions have been used to construct an approximate surrogate production function ${ }^{14}$, and they may also explain the existence and the stability in the factor markets of general equilibria of the "old" 19th-century neoclassical authors $^{15}$.

So there are reasons to return to Samuelson's reconstruction of neoclassical theory on the basis of linear wage curves - not in the sense of a rigorous theory that holds without exception, but as an approximation, this time not based on the labour theory of value, but on weaker and perhaps more plausible assumptions. Admittedly, linear wage curves are a fiction. Theory and empirical investigations leave no doubt that Wicksell-effects are ubiquitous, even if the deviation from linearity is, especially for numéraires consisting of many commodities, not large. The examination of this fiction remains nonetheless essential, for it is necessary for neoclassical theory to avoid declination and reverse capital deepening. We do, of course, not pretend that wage curves are linear, but we propose to return, temporarily at least, to the mental experiment of linear wage curves. This should be of interest also for readers who are not inclined to regard empirical wage curves as quasi-linear. As will be seen, the assumption is not essential for all of what follows. We shall argue in Section 5 that weaker assumptions suffice to preserve essential results.

Cutting a long story short, we now take up point (2) and assume strictly linear wage curves: what then about Samuelson's assumption that, the higher the labour productivity of an individual technique, the lower the corresponding maximum rate of profit? ${ }^{16}$ Samuelson seems to have taken it for granted that, by and large, for any given technique, the productivity of capital would be the lower, the higher the productivity of labour. If this is the case, we shall say that we have a spectrum of techniques with inverse productivities. That spectra are not always like that, follows from the following consideration: Joan Robinson thought that "good" techniques would be characterized by high productivities of both, of labour and of capital. If there is a really good technique, it dominates all others and there is no substitution. Samuelson's assumption implies that, as one moves down the envelope, all or most techniques will appear one by one. He gave a graphic example of techniques alpha, beta, gamma, delta, appearing in this order on the envelope, except that a process epsilon remained entirely below. 
The number of wage curves involved here can be very large. In our standard example, we have ten countries, each with 100 industries or sectors, producing the same commodities. Information is decentralized, in that producers know or can find out what the technique of each of their nine competitors in their industry is, while nobody knows the peculiarities of all industries in all countries. If we assume away any possible specificity of the capital goods or of combinations of processes, ten methods can in principle be used in each industry, so that there are in principle $10^{100}$ wage curves, and the question now is: how many of them will make it up on the envelope? Most of them, as Samuelson's construction seems to suggest - then Sraffa's formulation of a "rapid succession of switches" is appropriate - or only one, because there is only one dominant technique independently of distribution, as Joan Robinson thought? Both positions have intuitive appeal. The neoclassical might say: if the productivity of labour is high, the engineers have probably achieved this at the expense of a high productivity of capital. Joan Robinson might reply that research is always directed at finding methods that beat those of the competitors in a broad set of circumstances, that entrepreneurs aim at surplus profits without favouring the use of capital or labour, that sunk costs of finding new techniques are high and that therefore successful techniques are not numerous and do not easily change because of redistribution.

The central assumption now made is in a sense a compromise: The two productivities shall be random and completely independent of each other. More formally: we can order each finite set of techniques $\sigma=1 \ldots, s$, with wage curves $w^{\sigma}(r)$, such that the ordering corresponds to the productivities of labour and $w^{1}(0)>\cdots>w^{s}(0)$. The assumption then is that the ordering of the corresponding $R_{1}, \ldots, R_{s}$ of the maximum rates of profits or productivities of capital shall be independent of the ordering of the productivities of labour or that, which is an equivalent expression of the central assumption, all permutations of the $R_{\sigma}$ are equally likely. We thus write the ascending sequence of the maximum rates of profit as $R_{\sigma_{1}}, \ldots, R_{\sigma_{s}}$, where $\left(\sigma_{1}, \ldots, \sigma_{s}\right)$ is a permutation of $(1, \ldots, s) . R_{s}$ is the maximum rate of profit pertaining to wage curve $w^{s}(r) . R_{\sigma_{s}}$ is the largest among all $s$ maximum rates of profit.

One might investigate the plausibility of this assumption by empirical methods, but we leave this for future research ${ }^{17}$. Meanwhile it turns out that the assumption leads to a new mathematical problem: how many of these $s$ wage curves can be expected to appear on the envelope? The question seems not to have been asked before, except for a paper by Schefold (2013b), which gives an easy provisional answer. The complete solution, to be presented below, is more difficult. It has been found by Götz Kersting and has surprising implications.

We begin by summarizing Schefold's sketch of the matter. As we shall see, it leads to an upper bound, if we proceed "from above" and look at the wage curve with the highest productivity of labour, $w^{1}(r)$. It is on the envelope by definition, with $w^{1}(0)>w^{\sigma}(0) ; \sigma=2, \ldots, s$. Coming down from above, it is clear that $w^{2}(r)$ will be on the envelope with probability $\frac{1}{2}$, for it will be on the envelope if $R_{2}>R_{1}$ and it will be dominated by the first wage curve throughout if $R_{1}>R_{2}$. Both possibilities are equally likely by assumption (we assume all maximum rates of profit and all productivities of labour to be different). Continuing to look from above, we find that $w^{3}(r)$ appears on the envelope with probability $\frac{1}{3}$, for $R_{3}>R_{1}$ and $R_{3}>R_{2}$ is possible both with $R_{1}>R_{2}$ and $R_{2}>R_{1}$, hence in two cases out of six, hence with probability $\frac{1}{3}$. By induction, we find that the expected number of wage curves on the envelope, $\omega$, is given by $1+\frac{1}{2}+\frac{1}{3}+\cdots+\frac{1}{s}$. If $s$ is large, we can approximate $\omega$ by the natural logarithm:

$$
\omega=\ln s .
$$


The natural logarithm of $s$ tends to infinity, but slowly; the share of the wage curves, which appear on the envelope, $\Omega$, is given by $\Omega=\frac{\omega}{s}=\frac{\ln s}{s}$, and this tends to zero as $s$ tends to infinity; the number of efficient techniques becomes vanishingly small relatively to the number of potential techniques below the envelope.

It has been easy to derive this estimate, but it overstates the number of wage curves on the envelope, for the reason that a wage curve coming later, as one moves down on the envelope, can become dominant (appear on the envelope) by dominating one or even several earlier wage curves, which seemed to have become dominant by looking only from above. The possibility is illustrated in Diagram 1.

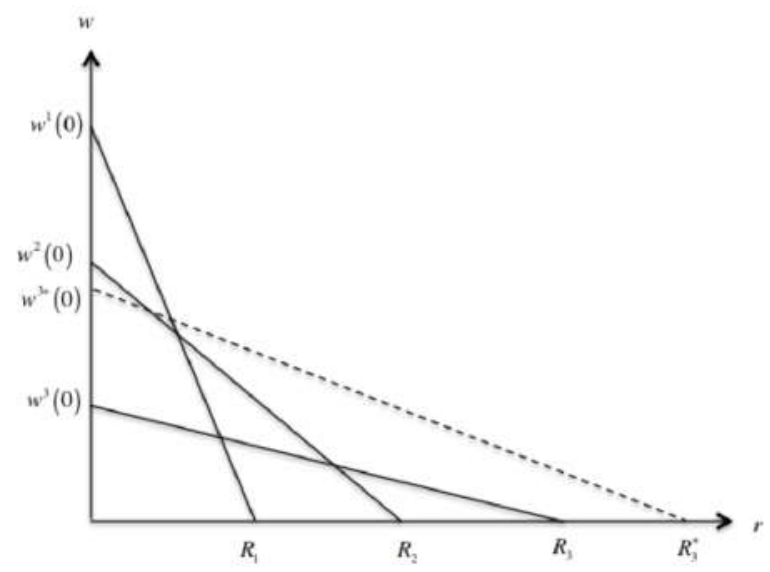

Diagram 1: Three wage curves with neoclassical ordering (the ordering of the maximum rates of profit is inverse to that of the wage rates). If the maximum wage of the third wage curve is higher (dotted line), one switch-point disappears (dominance from below).

The unbroken lines are three straight wage curves of the neoclassical type: the ordering of the productivities of capital is inverse to the ordering of the productivities of labour. But the dotted line shows a possible alternative to the third wage curve such that it dominates the second wage curve, which seemed to be on the envelope, when one looked from above and considered only the first two wage curves.

Because of the phenomenon of 'dominance from below', the formula $\omega=\ln s$ overstates the number of wage curves to be expected on the envelope, but by how much? This is a curiously tricky mathematical problem. It is clear that both $w^{1}(r)$ and $w^{\sigma_{s}}(r)$ can never be dominated from below, but a $w^{\sigma}(r)$, where $\sigma$ is "in the middle", can possibly dominate many wage curves with higher labour productivities from below at intermediate rates of profit, which might have been dominant, looked at from above. Yet, the formula $\omega=\ln s$ seemed to provide an only moderate overstatement according to empirical investigations, applying the formula to the results in Han and Schefold (2006), in Zambelli (2017) and also in unpublished numerical experiments. (More on Zambelli's results and ours at the end of the paper, Section 5, sixth point.) The numerical experiments were based on the assumption that the wage rates at $r=0$ and the maximum rates of profit were at equal distances (the wage curves were drawn in a regular grid). Looking at Diagram 1, one realises that the transition to a regular grid can make a difference: if $w^{3 *}(0)$ is shifted downwards, so as to obtain as much space between $w^{2}(0)$ and $w^{3 *}(0)$ as between $w^{1}(0)$ and $w^{2}(0)$, the dominance from below disappears. But dominance from below can evidently also occur, if the grid is regular. 


\section{Proposition 1}

Under the central assumption (equal probability of all orderings of the maximum rates of profit) and if $s$ straight wage curves are given, the expected number of wage curves on the envelope is equal to or lower than $\ln s$.

The empirical confirmations of Proposition 1 can only be approximate: real wage curves, as derived from empirical input-output tables, are not exactly straight; numerical experiments with straight wage curves are based on regular grids. Nonetheless, the empirical results are plausible.

The logarithm $\ln s$ tends to infinity with $s$, but slowly. In the standard example, with $10^{100}$ wage curves, the number of curves on the envelope is only $100 \ln 10 \cong 230$. The substitution possibilities are therefore far more limited than the enormous number of wage curves involved suggests. But we have found a much more radical result: there is virtually no change of the capital-labour ratio along the relevant range of the envelope, as $w$ becomes large, and the possibilities of substitution cluster in two small neighbourhoods: around the maximum wage rate $w^{1}(0)$ and the largest of the maximum rates of profit $R_{\sigma_{s}}$. The exact formulation of the proposition requires some preparation.

Let the wage curves $w^{1}(r), \ldots, w^{s}(r)$, with $w^{1}(0)>\cdots>w^{s}(0)$ (straight lines) of a spectrum of techniques be arranged in the rectangle $Q$, the wage curve box. The line segments $w^{\sigma}(r)$, defined by the end points $w^{\sigma}(0)$ and $R_{\sigma}$, can be represented by a point $P_{\sigma}$ in $Q$, with the end points as coordinates. $Q$ contains a grid, the horizontal lines of which are defined by $w^{\sigma}(0)$ and the vertical lines by the $R_{\sigma}$. We measure profit rates on the abscissa, which are pure numbers (pure numbers apart from the time dimension of interest, here taken into account via the length of the period of production). We measure on the ordinate output per head. When we look at the grid, these measures define the distances between the lines of the grid. The lines themselves may simply be numbered. Since the $w^{\sigma}(0)$ and the $R_{\sigma}$ are different, each horizontal and each vertical line will contain exactly one representative or anchor point $P_{\sigma}$, so that the grid in $Q$ looks like a perturbation matrix, except that $Q$ is not necessarily square and the grid is in general not regular, in that the distances between the grid lines vary. See Diagram 2, where we have five wage curves with the corresponding anchor points.

One easily confirms: Two (straight) wage curves like $w^{1}$ and $w^{2}$ in the wage curve box intersect inside the wage curve box if and only if the line segment $\overline{P_{1} P_{2}}$ is negatively sloped. Dominance occurs if and only if $\overline{P_{1} P_{2}}$ is positively sloped and $w^{1}$ and $w^{2}$ intersect at their endpoints on the boundary of $Q$, if and only if $\overline{P_{1} P_{2}}$ is horizontal or vertical.

Now we imagine that the wage curve box gets below the diagonal $d$ filled with more and more wage curves of number $s$, in such a way that all orderings of the maximum rates of profits are equally likely. We therefore stick to the central assumption adopted for Proposition 1 . The five wage curves drawn correspond to Samuelson's idea of a surrogate production function, but this picture will be destroyed if there is a wage curve dominating most of the envelope of $w^{1}, w^{2}, w^{3}, w^{4}$, such as a wage curve $w^{6}$ (dashed line, roughly parallel to diagonal $d$ ) and represented by point $P_{6}$. If one or several such points exist in the upper right corner of the box $A B F E$, say in the triangle $D F G$, a new envelope arises with - as will be shown - only a few switch-points except near the corners $E$ and $B$. As we have drawn the diagram, the envelope consists of short stretches of $w^{1}$ and $w^{4}$, and of the long stretch of $w^{6}$. Substitutions, which will take place on this envelope, will only occur near the corners $E$ and $B$, if there is only one such point $P_{6}$ in the triangle $D F G$. If there are several such points, the slopes of the corresponding wage curves will necessarily be almost equal, hence substitutions would (except at the corners) not lead to appreciable variations of the capital-labour ratio. 


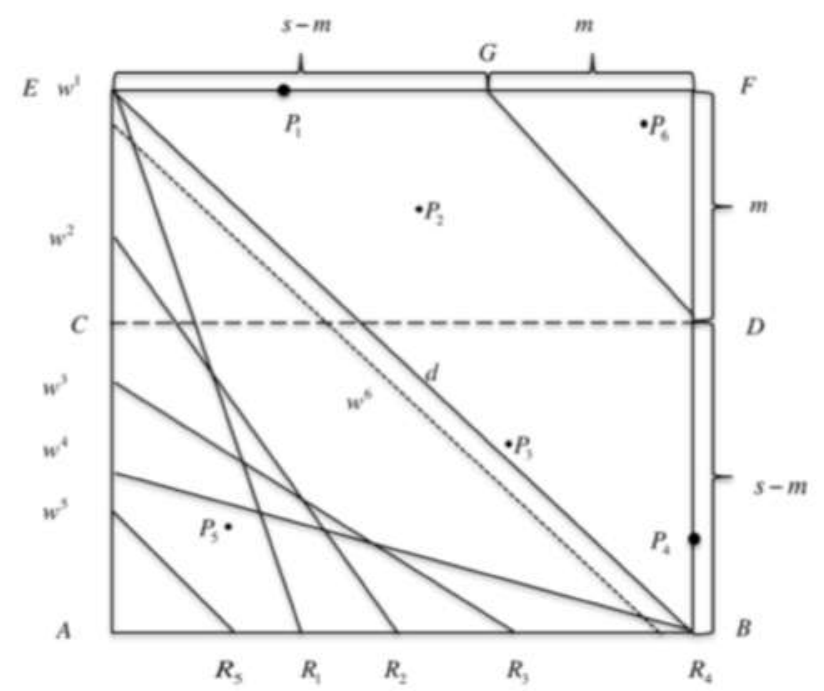

Diagram 2: The wage curves and their anchor by points in the wage curve box. One wage curve, $w^{5}$, is entirely dominated by the others, and such domination shows in the fact that $P_{1}, P_{2}, P_{3}, P_{4}$ are all above and to the right of $P_{5}$.

How likely is that to happen? In order to show it, we calculate the probability $\pi$ that it does not happen, that is, we calculate the probability $\pi$ that there is no anchor point in the triangle $D F G$; therefore that all $s$ anchor points lie in $A B D G E$. Triangle $D F G$ is on $m$ rows and $m$ columns, trapezium $C D G E$ is on $m$ lines with rows of lengths between $s-m$ and $s$. The point on the first row can therefore lie on one of $s-m$ places, the point on the second row (which, in trapezium $C D G E$, has $s-m+1$ places) can also lie on one of $s-m$ places, since one column has been occupied by the point on the first row. Similarly for $m-2$ later rows. The number of possible placements of points in the trapezium is $(s-m)^{m}$.

Since $m$ columns have been occupied in the trapezium, there remain $s-m$ columns to be occupied in the rectangle $A B D C$ with $s-m$ rows; $s-m$ points can be placed in the remaining rectangle $A B D C$ in $(s-m)$ ! ways.

The entire box $A B F E$ can be occupied in $s$ ! ways. Hence the probability $\pi$ we are looking for is, with $0 \leq m<s$, given by

$$
\pi=\frac{(s-m)^{m}(s-m) !}{s !} .
$$

Two special cases may be considered, before we transform this formula:

If $m=0$, trivially $\pi=1$.

If $m=1, \pi=\frac{s-1}{s}=1-\frac{1}{s}$ and $\bar{\pi}$, the probability that at least one point like $P_{1}$ is in the upper right corner of $Q$, is $\bar{\pi}=\frac{1}{s}$. This is the Joan Robinson case: One wage curve, the diagonal $d$, representing one best technique, dominates all others. This is unlikely, but the surprising result will be that the unlikely case becomes likely with only small modifications. 
In order to calculate the general case, it is convenient to begin with $1 / \pi$ :

$$
\begin{aligned}
\frac{1}{\pi} & =\frac{s !}{(s-m)^{m}(s-m) !}=\frac{s(s-1) \cdots(s-m+1)}{(s-m)^{m}} \\
& =\frac{s}{s-m} \cdot \frac{s-1}{s-m} \cdots \frac{s-m+1}{s-m} \\
& =\left(1+\frac{m}{s-m}\right)\left(1+\frac{m-1}{s-m}\right) \cdots\left(1+\frac{1}{s-m}\right)
\end{aligned}
$$

The ratios $m /(s-m), \ldots, 1 /(s-m)$ are small for large $s$, given $m$. Hence they may be approximated by using the formula $\ln (1+x) \cong x$, and the approximation will be exact, if we let $s$ and $m$ go to infinity, but in such a way that the ratio $m /(s-m)$ tends to zero. This is achieved by assuming ${ }^{18} m=\sqrt{\gamma s}$, where $1 \leq \gamma \leq s$, since we had assumed $m \leq s$. The coefficient $\gamma$ then is kept constant, as $s \rightarrow \infty$ :

$$
\begin{aligned}
\frac{1}{\pi} & =\exp \left[\ln \left(1+\frac{m}{s-m}\right)+\cdots+\ln \left(1+\frac{1}{s-m}\right)\right] \\
& \cong \exp \left[\frac{1}{s-m}(1+\cdots+m)\right]=\exp \left[\frac{m(m+1)}{2(s-m)}\right] \\
& \cong \exp \frac{\gamma s+\sqrt{\gamma s}}{2(s-\sqrt{\gamma s})}=\exp \frac{\gamma+\sqrt{\gamma / s}}{2-2 \sqrt{\gamma / s}} \stackrel{s \rightarrow \infty}{\longrightarrow} \sqrt{e^{\gamma}}
\end{aligned}
$$

We get

$$
\lim _{s \rightarrow \infty} \pi=\frac{1}{\sqrt{e^{\gamma}}}
$$

and $\bar{\pi}$ tends to

$$
\lim _{s \rightarrow \infty} \bar{\pi}=1-\frac{1}{\sqrt{e^{\gamma}}} .
$$

If $\gamma=1$, we thus find that the wage curves will be dominated by at least one wage curve very close to the diagonal $d$ in roughly one half of the cases ${ }^{19}$. And if we set a higher value for the constant $\gamma$, this will become virtually certain, as $\bar{\pi}$ will tend to 1 , without, however, reaching that limit $s$, since $\gamma$ must be kept bounded. We have proved:

\section{Proposition 2}

If the number $s$ of techniques can be increased indefinitely, to each probability $\pi^{*}, 0<\pi^{*}<1$, a number $\gamma$ can be assigned such that with probability $\pi^{*}$ there will be a wage curve $w^{*}$ with a distance to the diagonal $d$, measured horizontally and vertically, not larger than $m=\sqrt{\gamma s}$.

\section{Corollary 1}

The envelope of all wage curves will lie in between $w^{*}$ and $d$.

Note that wage curve $w^{6}$ in Diagram 2 illustrates such a $w^{*}$.

\section{Corollary 2}

The relative distance, bounded from above by $\mathrm{m} / \mathrm{s}=\sqrt{\gamma / \mathrm{s}}$, tends to zero. 
Will this distance also tend to zero, if we measure it as percentage points of rates of profit along the abscissa and in terms of output per head along the ordinate? This will evidently depend on how we model the increase in the number of techniques. It need not be the case, if additional techniques have higher output per head and higher rates of profit - the wage curve box then will grow larger -, but if additional techniques spring up in between those which are already there if growth of the box is bounded -, our grid becomes denser, not larger. Obviously:

\section{Corollary 3}

If additional techniques come in without an increase of maximum output per head and of the maximum of the rates of profit, such that the $R_{\sigma}$ cluster all along the abscissa and the $w^{\sigma}(0)$ along the ordinate, the distance between $w^{*}$ and $d$ tends to zero.

In order to observe the process of clustering for large, but finite $s$, we go back to Diagram 2 . As $s$ increases, additional techniques are brought in and the productivities of labour and capital increase, but not indefinitely in a finite world. So let us assume a given large $s$ - we may again think of our standard example with $10^{100}$ wage curves. We measure the wage rate and the rate of profit in relative terms by normalizing $w^{1}(0)=1$ and $R_{\sigma_{s}}=1$. We may impose a degree of certainty $c$ that there is at least one wage curve close to diagonal $d$ (corresponding to $w^{6}$ in Diagram 2) by requiring that $\pi \leq c$, so that $\sqrt{e^{\gamma}} \geq 1 / c$ or $\gamma \geq \ln \left(1 / c^{2}\right)$. If we demand that such a wage curve exists with the expectation of a trillion to one, we have $c=10^{-12}$ and $\gamma=\ln \left(1 / c^{2}\right) \cong 55$.

This defines $m=\sqrt{\gamma s}$; the wage rate $w^{6}(0)$ of this wage curve $w^{6}$ therefore is in an interval $I_{w}$ between a $\tilde{w}=1-\mathrm{m} / \mathrm{s}$ and $w^{1}(0)=1$. The maximum rate of profit $R_{6}$ is in an interval $I_{R}$ between a $\tilde{R}=R_{\sigma_{s}}-m / s$ and $R_{\sigma_{s}}=1$. In the standard example, we have $m \cong \sqrt{55} \cdot 10^{50}$. The length of the intervals $I_{w}$ and $I_{R}{ }^{20}$ is $\mathrm{m} / \mathrm{s} \cong 7 \cdot 10^{-50}$. If $s$ is larger than astronomical numbers in the standard example, $\mathrm{m} / \mathrm{s}$ is smaller than diameters of elementary particles relative to macroscopic terrestrial objects.

We now want to show that the intensity of capital on the envelope is, except at rates of profit very close to zero or to $R_{\sigma_{s}}$, very close to the intensity of capital of $w^{6}$, which is very close to the intensity of capital of the diagonal $d$, interpreted as a potential wage curve with intensity of capital $w^{1}(0) / R_{\sigma_{s}}$ (equal to one with our normalization). Indeed, it is geometrically obvious that $\frac{w^{6}(0)}{R_{6}} \cong \frac{w^{1}(0)}{R_{\sigma_{s}}}$ since $w^{6}(0)$ is in $I_{w}$ and $R_{6}$ is in $I_{R}$. If $w^{6}$ is not part of the envelope, the envelope itself is even closer to $d$.

We can summarize broadly as follows: Having fixed $\gamma$ in order to reach a desired degree of certainty, we can still increase $s$, if the technology is rich enough in methods, and in theory we can go with $s$ to infinity so as to bring the envelope, which consists of line segments, arbitrarily close to $d$, so that the capital-intensities, represented by the line segments, must approach the slope of $d$, since the envelope as a whole is monotonically falling.

\section{Proposition 3}

The intensity of capital tends, except in a vanishingly small neighbourhood of $r=0$ and $r=R_{s}$, to $w^{1}(0) / R_{\sigma_{s}}$, as s tends to infinity.

Matters look different for finite $s$, as one approaches the corners $E$ and $B$. The envelope may start at $E$ with a nearly vertical slope (the intensity of capital is large initially) and ends at $B$ with a tangentially horizontal slope (the intensity of capital may be small). It then seems to follow from our assumptions that the envelope has the properties of the wage curve one derives 
from a neoclassical production function, but only in the small, at small rates of profit or wage rates. In a world with a large, but finite $s$, the envelope could therefore look as in Diagram 3, consisting of a large number of line stretches.

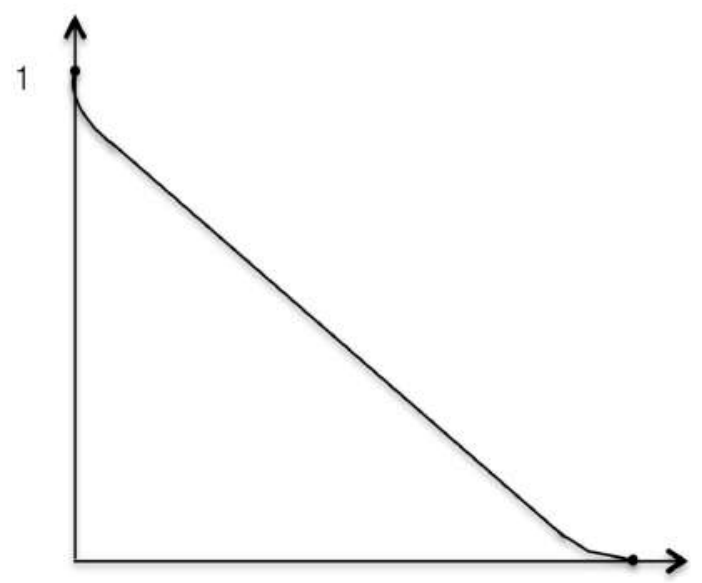

Diagram 3: The envelope for many techniques .

The absolute value of the slope of the first wage curve in going down from $E$ and of the last, when one arrives at $B$, is a priori anywhere between infinity and the slope of the diagonal, and between that and zero respectively, so that these initial and terminal wage curves determine the slopes of the beginning and the end of the envelope. The probability that the first wage curve is the steepest is the same as that it is already the dominating wage curve and actually equal to $d$, namely $1 / s$. Moreover, as $s$ increases, point $P_{6}$ will be driven into the upper right corner, as $\mathrm{m} / \mathrm{s}$ tends to zero. This means that the wage curve corresponding to $P_{6}, w^{6}$, will tend to the diagonal also near the axes, and the possible wage curves of high and low capital-intensity there will tend to get dominated so that the intensity of capital will, in our normalization, tend to one near both ends. There remains little room for the substitution of capital for labour with these assumptions, and Joan Robinson's position then is vindicated.

To assume a smooth wage curve as in Diagram 3 is a concession to neoclassical theory. Our original assumptions define an envelope composed of line segments of a subsets of the wage curves $w^{\sigma}(r)$. As one moves down the envelope, the wage rate falls monotonically as the rate of profit rises. Each line segment of a $w^{\sigma}(r)$ on the envelope is associated with a definite capital intensity $k_{\sigma}$, which falls as $r$ rises, and an output per head $y_{\sigma}=w^{\sigma}(0)$, which also falls. If one associates each of the capital intensities, going now from the lowest to the highest, with the corresponding output per head, $y_{\sigma}=w^{\sigma}(0)$, one obtains a rising step function $y_{\sigma}=f\left(k_{\sigma}\right)$, and the question is whether a smoothing of the envelope will lead to a smooth production function fulfilling the marginal productivity conditions.

We now postulate that a smooth envelope $w(r)$ can be given with stylized assumptions, that is with $w^{\prime}(0)=k_{\sigma_{s}} \gg 1, w^{\prime}\left(R_{\sigma_{s}}\right)$ small and, as in Diagram 3, with a long linear stretch in the middle between rates of profit $r_{1}$ and $r_{2}$, as an expression of the fact that the envelope approximates the diagonal of the wage curve box, like wage curve $w^{6}(r)$ in Diagram 2 according to what we have proved. The intensity of capital $k$ is equal to the absolute value of the slope of $w(r)$ at each $r$ and $y=f(k)$ is given by the intersection of the tangent with the ordinate so that $f(k)$ rises monotonically and continuously. The intensity of capital is equal to one along the 
linear segment of $w(r)$ between $r_{1}$ and $r_{2}$, if we assume a normalised wage curve box, and $f(1)$ is well defined, but $f(k)$ has a kink at $k=1$, since the rate of profit equals $r_{2}$, if we approach $k=1$ from below, and $r$ falls from $r_{1}$, as $k$ is raised beyond $k=1$. This indeterminacy of the marginal productivity condition is not small, since $r_{1}$ tends to zero and $r_{2}$ to $R_{\sigma_{s}}$, as $s$ increases.

To show this more formally, we have to go back to the well-known mathematical construction of the production function, starting from the wage curve (Samuelson 1962, p. 202; Schefold 1989, pp. 297-8; the procedure is the reversal of the derivation of the wage curve from the production function explained in Note 7).

For each individual technique we have output per head $y_{\sigma}=w^{\sigma}(0)$ because of the choice of the numéraire (Note 2). Accounting yields $\left(y_{\sigma}-w(r)\right) / r=k$, and the tangent to the wage curve $w(r)$ at $r$ has slope $-k_{\sigma}$, because $w(r)$ is the envelope of the wage curves. Hence $k=-w^{\prime}(r)$ at each $r$, and with this the production function can be defined parametrically by $f(k)=w(r)+r k=$ $w(r)-r w^{\prime}(r)$. Now $f(k)$ is well defined, if $w^{\prime \prime}(r) \neq 0$, which is the case in the intervals [0, $\left.r_{1}\right)$ and in $\left(r_{2}, R_{\sigma_{s}}\right]$, but $w^{\prime \prime}(r)=0$ in $\left[r_{1}, r_{2}\right]$. We have in $\left[0, r_{1}\right)$ and in $\left(r_{2}, R_{\sigma_{s}}\right]$ as in Note 7

$$
\frac{d f}{d k}=\frac{d f}{d r} / \frac{d k}{d r}=\frac{1}{-w^{\prime \prime}}\left(w^{\prime}-w^{\prime}-r w^{\prime \prime}\right)=r .
$$

This means that the production function, so defined, fulfils the first-order condition of the marginal productivity relationship (and similarly for the second order), but the linear section of $w(r)$, where the function $k=w^{\prime}(r)$ cannot be inverted, results in a kink of $f$ at $k=1$.

\section{Proposition 4}

Given the stylized assumptions of Diagram 3, an approximate surrogate production function $f(k)$ results, which fulfils the marginal productivity condition $f^{\prime}(k)=r$ and $f^{\prime \prime}<0$, except at $k=$ 1 , where the function is continuous, but the left-hand derivative equals $r_{2}$ and the right-hand derivative equals $r_{1}$.

The indeterminacy of the marginal product is not small, for we get from Proposition 3:

\section{Corollary}

As $s$ tends to infinity, $r_{2}$ tends to $R_{\sigma_{s}}=1$ and $r_{1}$ to zero.

Since the economy will tend to a state with $k=1$, distribution remains unexplained. The underlying reason will emerge in the next section, where it is shown that the economy does not only tend to a specific value of the capital-labour ratio, but that the number of techniques that are actually eligible is surprisingly small. This means that, if we do not flatten the envelope by introducing a continuum of techniques and by assuming, à la Samuelson, that it is as smooth as in Diagram 3, there will be substitution in the sense of changing of techniques at the (small number of) switchpoints, but there will be virtually no substitution in the (from the point of view of neoclassical theory) relevant sense of changes of the capital-labour ratios, for they tend to equality, except at the ends of the envelope, as will be shown more rigorously in the next section. This, then, is the new critique of neoclassical theory, which results on the basis of the Samuelsonian assumption that wage curves are linear, irrespective of whether one regards this assumption merely as a concession in the debate or because one regards it as licit in the belief that empirical wage curves are quasi-linear or because one argues that wage curves must tend to be quasi-linear as a result of the random character of systems. For extensions to the non-linear case, see the sixth point in Section 5. 


\section{Theorem by Kersting}

The theorem is introduced as a mathematical problem with its own notation. It may be studied independently of the other sections of the paper, but it provides an essential insight for the economic analysis, by which it is inspired.

Let $s \in \mathbb{N}$ and let $\left(\sigma_{1}, \ldots, \sigma_{s}\right)$ be a random permutation of $(1, \ldots, s)$. Denote by $w^{k}$ the straight line in the plane passing through the points $k$ at the ordinate and $\sigma_{k}$ at the abscissa. We are going to study their envelope $w$ in the plane's first quadrant,

$$
w(r):=\max _{1 \leq k \leq s} w^{k}(r), \quad r \in[0, s] .
$$

Between its endpoints $(0, s)$ and $(s, 0)$ it is made up of several line segments. Let $K$ be the set of

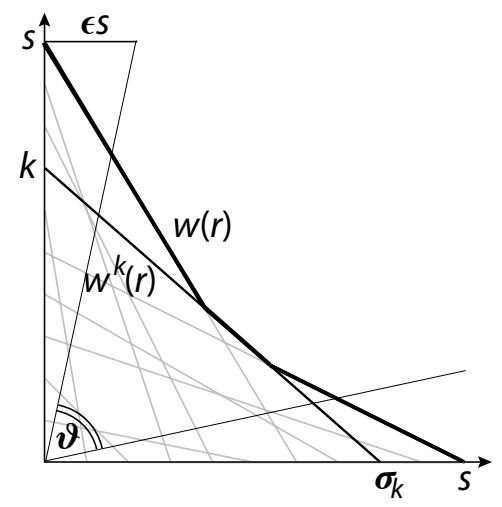

Diagram 4: The envelope with $s=10$.

points in the plane, where these segments join up (the "kinks" of $w$ ), and denote its cardinality by

$$
X_{s}:=\# K
$$

Also, for $0<\epsilon<1$ set

$$
X_{s, \epsilon}:=\#\{(i, j) \in K: \epsilon i \leq j \text { and } \epsilon j \leq i\},
$$

which is the number of kinks in the sector within the first quadrant with the angle $\vartheta=\pi / 2-$ $2 \arctan \epsilon$. Note that $X_{s}=X_{s, 1 / s}$.

Theorem. As $s \rightarrow \infty$

$$
E\left[X_{s}\right] \sim \frac{2}{3} \ln s \quad \text { and } \quad E\left[X_{s, \epsilon}\right] \sim \frac{2}{3} \ln \epsilon^{-1}
$$

The intuition behind this result is as follows: The envelope $w$ is primarily made up of a few lines $w^{k}$, which have the property that both $k$ and $\sigma_{k}$ are close to $s$ (the deviation being of order $\sqrt{s}$ ). They show up in the expectations $E\left[X_{s, \epsilon}\right]$, with increasing $s$ their number remains bounded. Additionally, $w$ contains at both ends lines $w^{k}$ where just $k$ or just $\sigma_{k}$ is close to $s$, including the lines with $k=s$ or $\sigma_{k}=s$. Our result indicates that there are on average $\frac{1}{3} \ln s$ many at each end. 
The next figure illustrates these asymptotic results by simulations from samples of size 10.000. The dots in the left-hand illustration specify the simulated expectations $E\left[X_{s}\right]$ with $s=10^{a}$, $a=1, \ldots, 9$, and the line is the function $\frac{2}{3} \ln s$. The dots in the right-hand graphics give the simulated expectations of $E\left[X_{s, \epsilon}\right]$ for the two values of $\epsilon$ when $\frac{2}{3} \ln \epsilon^{-1}$ is equal to 1 or 2 . They correspond to the angles $\vartheta$ with degrees 64.8 and 84.3 , respectively.
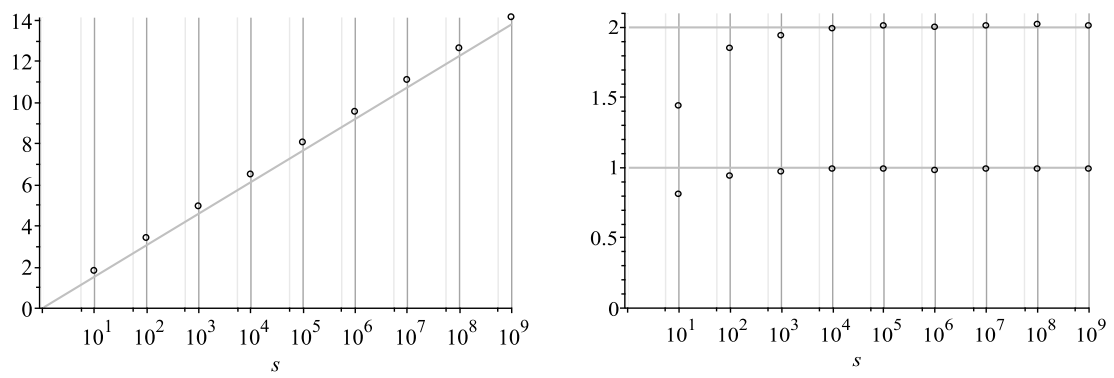

Diagram 5: The simulations.

(Outline of) Proof. The full proof will be given in a separate paper. Here we omit some technical details and focus on the central issues.

(i) Let us call a quadrupel $(a, b, c, d)$ of natural numbers a constellation, if $1 \leq a<b \leq s$ and $1 \leq c<d \leq s$. It determines the point $(i, j)$ of intersection of the straight line between

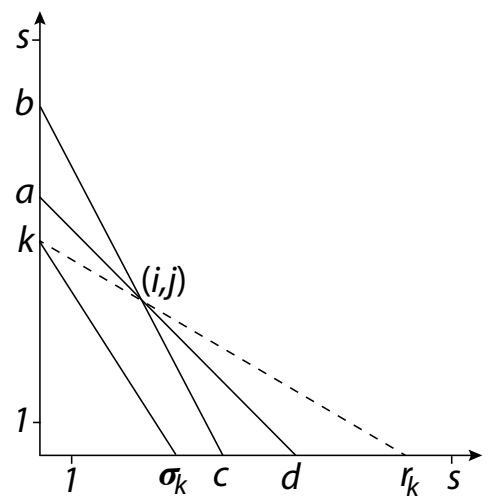

Diagram 6: A constellation.

$(0, a)$ and $(d, 0)$ and the line between $(0, b)$ and $(c, 0)$. Each element of $K$ is such an intersection point, which suggests to count $K$ by deciding for each intersection point, whether it belongs to $K$ or not. Thus, we assign to each constellation $(a, b, c, d)$ a random variable, indicating that it participates in the envelope in the sense that both lines from $a$ to $d$ and from $b$ to $c$ take part in $w$. In other terms:

$$
Y_{a b c d}:= \begin{cases}1, & \text { if } \sigma_{a}=d, \sigma_{b}=c \text { and }(i, j) \in K, \\ 0 & \text { else. }\end{cases}
$$

Each element of $K$ will be captured by one of the random variables. Some element of $K$ may be multiply recorded, since we have $(i, j)=\left(i^{\prime}, j^{\prime}\right)$ for some pairs of constellations, however, this 
feature is negligible in the limit $s \rightarrow \infty$. Summing these random variables over the constellations fulfilling $\varepsilon i \leq j \leq i / \varepsilon$ yields

$$
X_{s, \epsilon} \leq \sum_{\substack{a, b, c, d \\ \epsilon i \leq j \leq i / \epsilon}} Y_{a b c d}
$$

and the expectation taken on the left-hand side is well approximated by that on the right, that is

$$
E\left[X_{s, \epsilon}\right] \sim \sum_{\substack{a, b, c, d \\ \epsilon \leq j / i \leq 1 / \epsilon}} P\left(Y_{a, b, c, d}=1\right)
$$

as $s \rightarrow \infty$. To a certain extent this approximation is valid also for variable $\varepsilon$. In particular, inserting $\varepsilon=1 / s$ we obtain an approximation for the expectation of $X_{s}=X_{s, 1 / s}$.

(ii) Next we deduce a formula for the probability of $\left\{Y_{a b c d}=1\right\}$. The occurence of this event requires that none of the lines $w^{k}$ runs above the point $(i, j)$. This is immediate for $k \leq j$, and for $k>j$ it is required that $w^{k}(i) \leq j$. The latter condition boils after some algebra down to

$$
\sigma_{k} \leq r_{k} \quad \text { with } \quad r_{k}:=\frac{i k}{k-j} .
$$

The requirement has to be taken into account as long as $r_{k}<s$ or, equivalently, as long as

$$
k>\frac{j s}{s-i} .
$$

It follows that

$$
\left\{Y_{a b c d}=1\right\}=\left\{\sigma_{a}=d, \sigma_{b}=c \text { and } \sigma_{k} \leq r_{k} \text { for all } \frac{j s}{s-i}<k \leq s\right\} .
$$

Also observe that $r_{k}$ is increasing with decreasing $k$. Consequently, placing the lines $w^{s}, w^{s-1}$, ... one after the other, and checking all favorable and possible outcomes, we obtain the formula

$$
\begin{aligned}
P\left(Y_{a b c d}=1\right)=\frac{\left[r_{s}\right]}{s} \frac{\left[r_{s-1}\right]-1}{s-1} \cdots \frac{1}{b} \cdots \frac{1}{a} \cdots & =\frac{1}{a b} \prod_{\substack{j s \\
\frac{j s}{s-i}<k \leq s \\
k \neq a, b}} \frac{\left[r_{k}\right]-(s-k)}{s-(s-k)} \\
& =\frac{1}{a b} \prod_{\substack{\frac{j s}{s-i}<k \leq s \\
k \neq a, b}}\left(1-\frac{s-\left[r_{k}\right]}{k}\right)
\end{aligned}
$$

where $\left[r_{k}\right]$ denotes the biggest natural number not exceeding $r_{k}$. Incorporating in addition the inequalities $1-x \leq e^{-x}$ and $\left[r_{k}\right] \leq r_{k}$ yields

$$
P\left(Y_{a b c d}=1\right) \leq \frac{1}{a b} \exp \left(-\sum_{\substack{j s \\ \frac{j s}{s-i}<k \leq s \\ k \neq a, b}} \frac{s-r_{k}}{k}\right) .
$$

It can be shown that in our calculations we may substitute this upper bound for the probability. 
(iii) Now we derive an approximation for the right-hand expression in (3). From a heuristic point of view it is obvious that, with $s$ increasing, there are lines $w^{k}$ with the property that both $k$ and $\sigma_{k}$ don't deviate much from $s$. This has been shown more formally in Section 3 (Proposition 2 and Corollaries). This implies that the envelope $w$ is close to the diagonal connecting $(0, s)$ and $(s, 0)$. Therefore it is plausible, and indeed can be shown, that we may confine our considerations to constellations, fulfilling

$$
a, b, c, d \sim s
$$

as $s \rightarrow \infty$. In order to use these asymptotics for $i$ and $j$ we introduce the notations

$$
x:=b-a, y:=d-c, s-b:=u, s-d:=v .
$$

A quick calculation results in

$$
i=\frac{c d(b-a)}{c(b-a)+b(d-c)} \sim \frac{s x}{x+y}, j=\frac{a b(d-c)}{c(b-a)+b(d-c)} \sim \frac{s y}{x+y},
$$

hence

moreover

$$
s-i \sim \frac{s y}{x+y} \sim j, s-j \sim \frac{s x}{x+y} \sim i
$$

$$
s-i-j=\frac{c(b-a)(s-d)+b(d-c)(s-a)}{c(b-a)+b(d-c)} \sim \frac{x v+y u+x y}{x+y} .
$$

Coming back to (3) observe that for $k \leq s$ fulfilling (2) we have $k \sim s$. Consequently

$$
\frac{s-r_{k}}{k}=\frac{s-i}{k(k-j)}\left(k-\frac{j s}{s-i}\right) \sim \frac{s-i}{s(s-j)}\left(k-\frac{j s}{s-i}\right) \sim \frac{y}{s x}\left(k-\frac{j s}{s-i}\right)
$$

and

$$
\begin{aligned}
\sum_{\frac{j s}{s-i}<k \leq s} \frac{s-r_{k}}{k} & \sim \frac{y}{s x} \sum_{\frac{j s}{s-i}<k \leq s}\left(k-\frac{j s}{s-i}\right) \\
& \sim \frac{y}{s x} \int_{\frac{j s}{s-i}}^{s}\left(z-\frac{j s}{s-i}\right) d z \\
& =\frac{y}{s x} \frac{1}{2}\left(s-\frac{j s}{s-i}\right)^{2} \\
& =\frac{s y(s-i-j)^{2}}{2 x(s-i)^{2}} \\
& \sim \frac{f(x, y, u, v)}{2 s}
\end{aligned}
$$

with

$$
f(x, y, u, v):=\frac{(x v+y u+x y)^{2}}{x y}, x, y \geq 1, u, v \geq 0 .
$$

Applying the approximations to (3) (and neglecting the requirement $k \neq a, b$ for the summation index) yields

$$
P\left(Y_{a b c d}=1\right) \sim \frac{1}{s^{2}} \exp \left(-\frac{f(x, y, u, v)}{2 s}\right) .
$$


To arrive at the expectation these terms have to be summed for all constellations $(a, b, c, d)$, that is for all natural numbers $x, y \geq 1$ and $u, v \geq 0$ with $x+u<s, y+v<s$. Again it can be shown that the latter requirements can be ignored in the limit $s \rightarrow \infty$. Also in view of (4) we replace the condition $\varepsilon \leq j / i \leq 1 / \varepsilon$ by $\varepsilon \leq y / x \leq 1 / \varepsilon$. With these adjustments (1) yields

$$
E\left[X_{s, \varepsilon}\right] \sim \frac{1}{s^{2}} \sum_{\substack{x, y \in \mathbb{N}, u, v \in \mathbb{N}_{0} \\ \varepsilon \leq y / x \leq 1 / \varepsilon}} \exp \left(-\frac{f(x, y, u, v)}{2 s}\right)
$$

and also

$$
E\left[X_{s, \varepsilon}\right] \sim \frac{1}{s^{2}} \iiint \int_{\substack{x, y, u, v>0 \\ \varepsilon \leq y / x \leq 1 / \varepsilon}} \exp \left(-\frac{f(x, y, u, v)}{2 s}\right) d x d y d u d v .
$$

(iv) It remains to determine this fourfold integral. First we accomplish the integration with respect to $u$ and $v$ for fixed $x$ and $y$. To this end we introduce new coordinates

$$
z:=\frac{x v+y u+x y}{\sqrt{x y}}, w:=x u-y v .
$$

The condition $u, v \geq 0$ translates into

$$
z \geq \sqrt{x y} \quad, \quad-\frac{y}{x}(z \sqrt{x y}-x y) \leq w \leq \frac{x}{y}(z \sqrt{x y}-x y),
$$

and for the Jacobian determinant we have $\left|\frac{\partial(z, w)}{\partial(u, v)}\right|=-\left(x^{2}+y^{2}\right) / \sqrt{x y}$, thus $d u d v=\sqrt{x y} d z d w /\left(x^{2}+\right.$ $\left.y^{2}\right)$. Hence

$$
\begin{aligned}
\iint_{u, v>0} \exp ( & \left.-\frac{(x v+y u+x y)^{2}}{2 s x y}\right) d u d v \\
& =\int_{\sqrt{x y}}^{\infty} \int_{-\frac{y}{x}(z \sqrt{x y}-x y)}^{\frac{x}{y}(z \sqrt{x y}-x y)} e^{-z^{2} /(2 s)} \frac{\sqrt{x y} d w d z}{x^{2}+y^{2}} \\
& =\int_{\sqrt{x y}}^{\infty}\left(\frac{x}{y}+\frac{y}{x}\right)(z \sqrt{x y}-x y) e^{-z^{2} /(2 s)} \frac{\sqrt{x y}}{x^{2}+y^{2}} d z \\
& =\int_{\sqrt{x y}}^{\infty}(z-\sqrt{x y}) e^{-z^{2} /(2 s)} d z .
\end{aligned}
$$

Next we complete the calculation by performing the intergration with respect to $x$ and $y$. Here a second change of coordinates is helpful. We set $\eta=x y, \xi=y / x$ with corresponding Jacobian 
determinant $\left|\frac{\partial(\eta, \xi)}{\partial(x, y)}\right|=2 y / x=2 \xi$. Thus $d x d y=d \eta d \xi / 2 \xi$ and

$$
\begin{aligned}
\iiint \int_{\substack{x, y, u, v>0 \\
\varepsilon \leq y / x \leq 1 / \varepsilon}} \exp (- & \left.\frac{(x v+y u+x y)^{2}}{2 s x y}\right) d x d y d u d v \\
& =\iint_{\substack{x, y>0 \\
\varepsilon \leq y / x \leq 1 / \varepsilon}} \int_{\sqrt{x y}}^{\infty}(z-\sqrt{x y}) e^{-z^{2} /(2 s)} d z d x d y \\
& =\int_{\varepsilon}^{1 / \varepsilon} \int_{0}^{\infty} \int_{\sqrt{\eta}}^{\infty}(z-\sqrt{\eta}) e^{-z^{2} /(2 s)} d z \frac{d \eta d \xi}{2 \xi} \\
& =\int_{\varepsilon}^{1 / \varepsilon} \frac{d \xi}{2 \xi} \int_{0}^{\infty} e^{-z^{2} /(2 s)} \int_{0}^{z^{2}}(z-\sqrt{\eta}) d \eta d z \\
& =\ln \varepsilon^{-1} \int_{0}^{\infty} \frac{1}{3} z^{3} e^{-z^{2} /(2 s)} d z .
\end{aligned}
$$

By means of the subsitution $z^{\prime}=z^{2} /(2 s)$ it follows that the right-hand integral has the value $2 s^{2} / 3$. Going back to (5) we obtain

$$
E\left[X_{s, \varepsilon}\right] \sim \frac{2}{3} \ln \varepsilon^{-1},
$$

which is one of our claims. It holds also true, if we replace $\varepsilon$ by $1 / s$, which gives the other claim.

\section{$5 \quad$ Reconsidering results and assumptions}

The unusual findings at which we have arrived will provoke doubts and discussions. We shall try to respond to some potential objections. Before doing so, we synthesize some earlier results in a concrete economic example. Most economists do not take the production function for an immediate image of reality, but as a construction, which helps to visualize some aspects of what they perceive to be the basic characteristics of the economy: the production function illustrates the substitution of capital for labour, as labour becomes scarce and capital is accumulated in the long run or the substitution of labour for capital, if there is unemployment. In either case, substitution means that capital goods are recombined in different techniques - new ones in the process of growth, old ones, if unemployment is to be absorbed - and the nominal quantity of capital can - roughly - stay constant in the transformation. This happens in the relevant range of the rate of profit, and profit maximisation implies that the techniques chosen are on the envelope and efficient.

Diagram 7 shows how the substitution possibilities present themselves in our model. Suppose the industrial rate of profit is between $r_{1}$, say $3 \%$, and $r_{2}$, say $20 \%$, and the assumptions of the theorem of Section 4 hold. According to the earlier propositions, the envelope will be so close to the diagonal, that the distance between them may be neglected. This made it possible to conclude that the capital-labour ratio cannot change much on the envelope, except at its ends. Thanks to the Theorem, we can now turn to the change of techniques itself and calculate the number of substitutions (switch-points) that can be expected between $r_{1}$ and $r_{2}$ by calculating the difference between the numbers of switch-points of the larger cone with angle $\vartheta_{1}$, defined by 
$r_{1}$, and the narrower cone with angle $\vartheta_{2}$, defined by $r_{2}$, in Diagram 7 . For reasons of symmetry, one half of the difference must be taken.

The corresponding $\varepsilon_{1}$ and $\varepsilon_{2}$ result from the elementary intercept theorem as $\varepsilon_{i}=r_{i} /\left(1-r_{i}\right)$, assuming that we have the square wage curve box with side length 1 and a regular grid. The expected number of switch-points between $r_{1}$ and $r_{2}$ then is

$$
\frac{1}{2}\left(\frac{2}{3} \ln \frac{1}{\varepsilon_{1}}-\frac{2}{3} \ln \frac{1}{\varepsilon_{2}}\right)=\frac{1}{3}\left(\ln \left(\frac{1}{r_{1}}-1\right)-\ln \left(\frac{1}{r_{2}}-1\right)\right) .
$$

With $r_{1}=3 \%$ and $r_{2}=20 \%$ one gets $2.08 / 3$. Hence less than one switch-point, hence only one technique is expected to be encountered in this fairly large relevant range of the rate of profit. The capital-intensities of neighbouring techniques will for large $s$ nearly be the same in a much larger range, but here, there is not even a method change, and this despite the fact that, the number of techniques between zero and $r_{1}$ will tend to infinity as $s \rightarrow \infty$. The result is perplexing: as more techniques become available, fewer will appear in the relevant range, since most switch-points are pushed into the corners. The economy will stay at $k=1$, if we represent it by means of an approximate surrogate production function (Proposition 4), and it is confirmed that output per head will be near one, as long as the rate of profit remains in the relevant range.

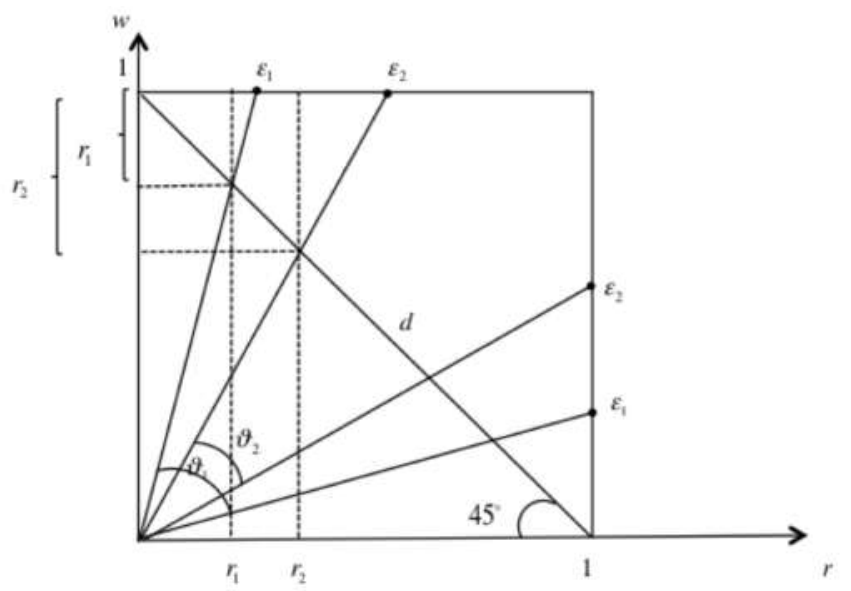

Diagram 7: Two cones (angles $\vartheta_{1}$ and $\vartheta_{2}$ ) defined by $r_{1}$ and $r_{2}$. The envelope within the difference of the cones between $r_{1}$ and $r_{2}$ is likely to contain only one technique with $r_{1}=3 \%$ and $r_{2}=20 \%$.

We now turn to six possible objections.

First,

one may object to our model that the distances between the grid lines need not shrink uniformly to zero, as $s$ tends to infinity, contrary to what is postulated in Section 4. If one excludes or ignores totally inefficient techniques and no such techniques exist in the set of techniques under consideration, there may for instance be a finite lower limit to the maximum rates of profit larger than the relevant range of the profit rates so that ample substitution possibilities might arise in that interval, as $s$ grows. However, as $s$ grows, there also grows the probability that an almost universally dominating technique of the type of wage curve $w^{6}$ in Diagram 2 will arise according to our central hypothesis, and except for very small rates of profit, the envelope will again be close to the diagonal. 
Second.

Hence we must reconsider the central hypothesis itself. We limit ourselves to the discussion of Proposition 1 and the domination from above. There is a large number $s$ of techniques with by assumption $w^{1}(0)>\cdots>w^{s}(0)$. We found $P\left(R_{\sigma}>R_{\sigma-1}, \ldots, R_{\sigma}>R_{1}\right)=1 / \sigma$; this holds, if we put $R_{0}=0$, for $\sigma=1, \ldots, s$. Now we ask whether our conclusions are affected substantially, if we assume that the die is loaded somehow in favour of the neoclassical assumption, in that a lowering of $w^{\sigma}(0)$ the transition from $w^{\sigma-1}(0)$ to $w^{\sigma}(0)$ - tends to lead to $R_{\sigma}>R_{\sigma-1}$. Countless variations of this idea can be imagined; let us consider some simple possibilities.

If we put $P\left(R_{\sigma}>R_{\sigma-1}, \ldots, R_{\sigma}>R_{1}\right)=\frac{\lambda}{\sigma}, \sigma=2, \ldots, s$, we get for the estimate from above for the expected number of wage curves on the envelope, $\omega$, now $\omega \cong \lambda \ln s$ : no significant improvement from the point of view of neoclassical theory.

An interesting possibility is to postulate $P\left(R_{\sigma}>R_{\sigma-1}, \ldots, R_{\sigma}>R_{1}\right)=\frac{1}{\sigma^{\beta}}, \beta>0, \beta \neq 1$; $\sigma=1, \ldots, s$. The case $\beta=1$ is what we have been discussing in Section 3 . If $\beta \neq 1$, one obtains

$$
\omega \cong \int_{1}^{s} \frac{1}{x^{\beta}} d x=\left.\frac{x^{1-\beta}}{1-\beta}\right|_{1} ^{s} .
$$

Only for completeness we mention that one gets $P\left(R_{\sigma}>R_{\sigma-1}, \ldots, R_{\sigma}>R_{1}\right)<1 / \sigma$ for $\beta>1$ and, for large $s, \omega \cong 1 /(\beta-1)$, for instance with $\beta=3 / 2, \omega \cong 2$, which means that there is virtually no substitution to be expected.

If $0<\beta<1, \omega \cong(1 /(1-\beta))\left(s^{1-\beta}-1\right)$, hence for $\beta=1 / 2 \omega \cong 2(\sqrt{s}-1)$. We now do get more techniques on the envelope, but still $\Omega=\omega / s \rightarrow 0$ as $s \rightarrow \infty$; the set of techniques appearing on the envelope remains infinitesimal relative to the set of all techniques. What is more important: the possibility that a $w^{\sigma-1}$ associated with a small $R_{\sigma-1}$ is followed by a $w^{\sigma}$ with a $R_{\sigma}$ close to $R_{\sigma_{s}}$ is not only not excluded by the assumption, but it becomes increasingly probable with rising $s$ even for some small $\sigma$. In other words, there will in this case as well appear anchor points in the upper right corner of the wage curve box in Diagram 2, only more slowly, and this means that almost universally dominant techniques will exist in this case as well and the envelope must approach the diagonal $d$ with very large $s$.

Finally, what about the case $\beta=0$ ? If all techniques are to appear on the envelope, the order of the maximum rates of profit must obviously be exactly inverse to that of output per head; one then must have that $w^{1}(0)>\cdots>w^{s}(0)$ implies $R_{1}<\cdots<R_{s}$. These relationships hold for the wage curves on the diagram for the envelope; they are then the result of the choice of efficient techniques; the wage curves are disentangled. To assume these relationships instead of deriving them would not mean to prove the existence of the neoclassical production function; it means simply to postulate it.

Third.

Would it be a legitimate way out for neoclassical theory to exclude the existence of almost universally dominating ('best') techniques on technical grounds? We can express this by looking at the chain of anchor points, which stand each for a segment of the envelope in Diagram 2. We have learnt in the upper right corner at $F$ we find the sparse points representing 'best' techniques. A technical assumption that excludes such techniques means that points in that neighbourhood would be prohibited. But such techniques may result from the combinations of processes taken from existing techniques, and are therefore not so easy to rule out in a non-trivial manner; an envelope of $10^{100}$ wage curves has not yet been calculated. Nonetheless, we can represent the exclusion of 'best' techniques in our framework by the assumption that for some given large $s$ and a not very large $m$ the triangle in Diagram 2 is empty. Since the number of switch-points 
and techniques in triangle $D F G$ is quite small according to the Theorem, it will remain true that the expectation of the number of switch-points and techniques is given by $\ln s$, if we stick to the central assumption, with this technological restriction, and the proof of Proposition 1 remains the same, as the reader can confirm. This assumption of the exclusion of 'best' techniques means that the envelope of the anchor points will loose its sharp bend, that the envelope of the techniques themselves ceases to be dominated by techniques close to the diagonal like $w^{6}(r)$ in Diagram 2 and becomes more convex so that the approximate surrogate production function also gets a softer bend instead of the kink of Proposition 4. The exclusion of 'best' techniques thus implies a step in the direction of neoclassical theory, and we note that a 'best' technique is in fact not visible in Zambelli's (2017) diagram, but it remains true that the number of substitution possibilities is limited there. We stick to our original central assumption without the technological restriction in the remainder of the paper, however, and leave the analysis of restricted technologies for a future investigation. It would have to be conducted at a different level of abstraction, for the absence of best techniques might result in practice from a not unlikely failure of the process of competition to reach the most efficient technique on the frontier (see also fifth point below).

\section{Fourth.}

The historical tendency, described by classical and neoclassical economists and visible in historical statistics, shows a predominant gain of the productivity of labour, made possible through a rise of the use of capital goods in changing proportions, but, in price terms, roughly in step with the rise of output and income. The neoclassicals explain the process by the scarcity of labour relative to the producible capital goods, the classical authors described the same phenomena as facts (mechanization), and Marx interpreted them as class struggle: the rearrangement of the labour power strengthens the capitalist. These stylized facts have been described as 'different forms of technical progress' (Schefold 1976); they also may be represented as a restricted technology. Why does this historical sequence, which has much truth in it, whatever the explanation, not manifest itself in a neoclassical production function, in which the historical rise of the capital-labour ratio at a virtually constant capital-output ratio is translated into a logical sequence ordered along the axis of the rate of profit: when it is high, as in the past, the intensity of capital is low, and the intensity is the higher the lower the rate of profit and the higher the wage rate?

Instead of arguing here about the theory of distribution or going into the methodological discussion about logical and historical time, I propose a very simple technical argument: in a large spectrum, those techniques that have a high productivity of capital (a large maximum rate of profit) also have on average a high productivity of labour. The maximum rate of profit rises, if the coefficients of $\mathbf{A}$ fall, since $\operatorname{dom} \mathbf{A}$ rises strictly monotonically with each element $a_{i j}$ of $\mathbf{A}$, but this also means that the productivity of labour increases, for this has been defined as $w(0)=\mathbf{d p} / \mathbf{q} \mathbf{l}$, with $\mathbf{d} \mathbf{p}=1$ and $\mathbf{q} \mathbf{l}=\mathbf{d}(\mathbf{I}-\mathbf{A})^{-\mathbf{1}} \mathbf{l}$. If any $a_{i j}$ falls, all the components of $\mathbf{q}$ fall and $w(0)$ rises, given $\mathbf{l}$. Hence, in the comparison of techniques, those that are 'good' because of a high $R$, will also tend to be 'good' because of a high $w(0)$. This, it seems to me, is a simple and intuitive explanation, why Joan Robinson made a good guess as to the possibilities of substitution on the envelope and why 'good' techniques cannot easily be assumed away. There is very little substitution among the techniques existing at any one point in time, but, as time goes on, mechanization rises. The rise of the intensity of capital is moderate, because progress lowers also the prices of capital goods, and the capital-output ratio can remain unaffected. The process of mechanization over time and the dominance of essentially one technique on the envelope do not contradict each other (see Diagram 8). 
Fifth.

The main empirical data we have to map processes of production are the coefficients of inputoutput tables. According to the theory, the methods used in different countries should be the same in each industry in which international competition is strong, but we note at best similarities. Also, if one calculates the envelope of the wage curves for different countries, represented by their input-output tables, one finds that no country is on that efficiency frontier, but at best near it (Zambelli 2017). We have abstracted from these phenomena with diverse causes, as we have here abstracted from the non-linearity of the wage-curves. These discrepancies between theory and facts are real, but, if the results are used with good judgement, they are not more damaging to the theory than is the observation of imperfections in virtually all markets to the theory of competition. - So much for the objections to the central assumption.

An important difference between this critique of the neoclassical approach and the older one, based on reswitching and reverse capital deepening, should be noted. Reswitching and reverse capital deepening are rare, as we saw by means of references to earlier work (Section 1) both empirically and, for large systems, in theory. Almost universally dominating techniques also are rare: rare in the set of all techniques. But while it does not matter much - it probably will not even get noticed - when reverse capital deepening occurs in reality, almost universally dominating techniques are reached or approximated through profit maximisation and show in the fact that actual techniques change through progress, not distribution.

Where did the idea of substitution as the central mechanism regulating distribution come from? The idea of a process of accumulation accompanied by a rising capital-labour ratio was, as we just have recalled, suggested by the rise of machinery in Ricardian times. Since there was labour-saving technical progress, one could also conceive of the opposite: to opt for less capitalintensive techniques to employ more labour, and factor prices would steer these processes. The principle of the process of substitution had been well understood in the case of land and labour thanks to the Ricardian theory of intensive rent. But capital is not land. It was found that the gain in labour productivity lowered not only the cost of output in real terms, but also that of capital goods so that the capital-output ratio could stay constant, which is the inverse of the maximum rate of profit. Accumulation could proceed at a constant rate of profit $\bar{r}$ and with constant shares of profits and wages, which means formally that the wage curve turns around the point of the maximum rate of profit $r=R$. Diagram 8 illustrates these 'stylized facts'. ${ }^{21}$ The wage curves shown are those of the universally dominating techniques of each period. A change in the rate of profit - whatever causes the change of distribution - does not lead to a substitution with significant changes of the capital-labour ratio. The latter changes only in the long run through technical progress.

One could summarize this last result by stating that our approach provides a theory of capital that can serve as the foundation for a Kaldorian theory of growth.

Sixth.

It remains to indicate how the exposition of this paper can be extended to take account of nonlinear wage curves. The reader may have noticed that the results of Proposition 1 hold also if any two wage curves that appear on the envelope intersect only once, at a switch point on the envelope. This assumption will be fulfilled, if reswitching and reverse capital deepening can be ruled out, following the Appendix in Schefold (2016), that is, if the dimension of the number of commodities in the system is sufficiently large and a boundedness condition holds. If the wage curves that appear on the envelope intersect at most once, we call them disentangled. Since this may be expected, Proposition 1 is therefore general and does not depend on the linearity of the wage curves in an essential way. 


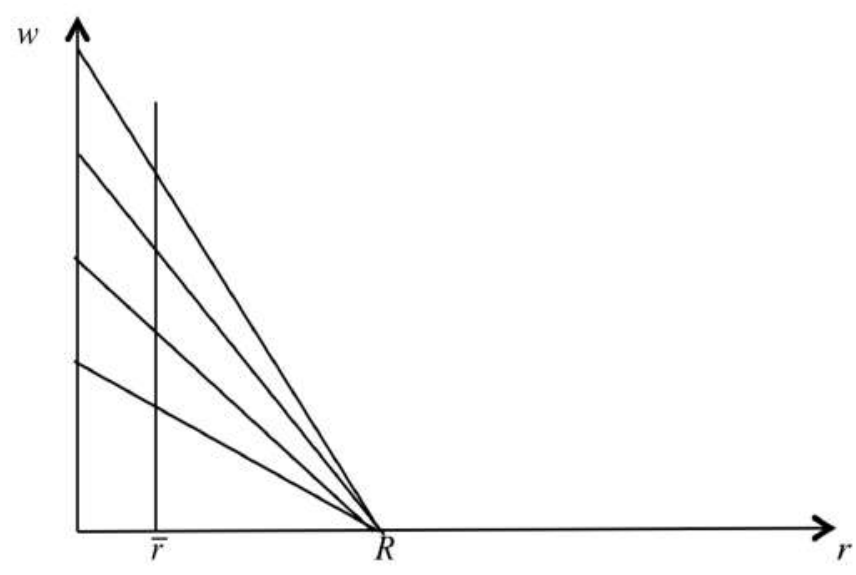

Diagram 8: A growing economy; the dominating technique of each period is represented by a wage curve showing the growth of the capita-labour ratio at a maximum rate of profit (inverse of the capital-output ratio) that stays constant..

Maintaining the central assumption and supposing wage curves that have no inflection points and intersect only once on the envelope, we can make a statement about the distribution of the switch points on the envelope. It is, however, weaker than the one contained in the Theorem of Section 4. As in the proof of Proposition 1, we estimate an upper limit $\omega$ for the number of switch points by looking at the envelope 'from above', but we now divide the procedure in two steps, adding up to $\omega^{\prime}$ the probabilities that $w^{1}, \ldots, w^{\vartheta s} ; 0<\vartheta<1$; is on the envelope; $\omega^{\prime \prime}$ is the sum of the remaining probabilities up to $s$. Hence

$$
\begin{aligned}
\omega & =\omega^{\prime}+\omega^{\prime \prime} \cong \ln s \\
\omega^{\prime} & =1+\frac{1}{2}+\frac{1}{3}+\cdots+\frac{1}{\vartheta s} \cong \ln (\vartheta s)=\ln s+\ln \vartheta \\
\omega^{\prime \prime} & =\omega-\omega^{\prime} \cong-\ln \vartheta=\ln \frac{1}{\vartheta}
\end{aligned}
$$

It follows that a tendentially infinite number of wage curves that appear on the envelope will start from the ordinate at $w^{1}(0), \ldots, w^{\vartheta s}(0)$, while only a finite number will make it on the envelope of the infinite number of wage curves $w^{\vartheta s+1}, \ldots, w^{s}$, as $s$ tends to infinity. A symmetric argument can be made by analysing how the wage curves start from the abscissa. By varying $\vartheta$, one finds that the wage curves on the envelope cluster at $r=0$ and at $r=R_{\sigma_{s}}$. By contrast, there is only a finite number of wage curves appearing on the envelope with $w^{\sigma}(0) \in(0, \vartheta s)$ and/or $R_{\sigma} \in\left(0, \vartheta R_{\sigma_{s}}\right)$. It is remarkable that this number depends only on $\vartheta$, not on $s$. The wage curves appearing on the envelope can therefore cluster only at the ends. Wage curves starting near the ends are likely to appear in the middle part of the envelope, if neoclassical Wicksell effects are sufficiently weak. The paradox remains: the more techniques there are, the shorter the envelope.

We cannot prove that the number of techniques used in the relevant range of the rate of profit is small without making assumptions about Wicksell effects, but if we do, we can show that the intensity of capital changes little in the middle part of the envelope ${ }^{22}$. To this extent, the main economic results of the paper hold, if wage curves are not straight ${ }^{23}$. 


\section{Notes}

${ }^{1} \mathrm{~A}$ technique is here usually given by a semi-positive, productive, indecomposable input-output matrix $\mathbf{A}$ for the production of $n$ goods, $\mathbf{A}=\left(a_{i j}\right)$, where $a_{i j}$ denotes the amount of commodity $j$ used for the production of a unit of commodity $i$ (industries on the rows, following Sraffa's notation). Each industry $i$ uses labour $l_{i}$; the labour vector is $\mathbf{l}$. There is a uniform rate of profit or interest $r$, a uniform wage rate $w$; the economy is in a stationary state, the wage is paid ex post, and prices are given by

$$
\mathbf{p}=(1+r) \mathbf{A} \mathbf{p}+w \mathbf{l}=w(\mathbf{I}-(1+r) \mathbf{A})^{-1} \mathbf{l} .
$$

${ }^{2}$ There is a vector $\mathbf{d}$; it represents the basket of goods, of which the net product is composed. This vector is also used as the numéraire, hence $\mathbf{d} \mathbf{p}=1$. Prices and the wage rate are then determined as functions of $r, \mathbf{p}(r)$ and $w(r)$. The wage curve $w(r)$ is monotonically falling between $r=0$ and a maximum rate of profit $R$, where $w=0$. If $\hat{\mathbf{p}}=\mathbf{p} / w$ are prices in terms of the wage rate, one has $1=\mathbf{d} \mathbf{p}=\mathbf{d} \hat{\mathbf{p}} w$, hence $w=1 / \mathbf{d} \hat{\mathbf{p}}$.

${ }^{3}$ We denote output per head by $y=\mathbf{d p} / \mathbf{q} \mathbf{l}$, $\mathbf{q}$ being the activity levels to produce $\mathbf{d}$ :

$$
\mathbf{q}(\mathbf{I}-\mathbf{A})=\mathbf{d} .
$$

Since all income goes to wages at $r=0$, we have $y(0)=w(0)$. Since output is the numéraire, $\mathbf{d p}=1$ and $y(r)=w(0)=\mathbf{d} \mathbf{p} / \mathbf{q} \mathbf{l}=1 / \mathbf{q} \mathbf{l}$ is independent of $r$, and $y=r k+w, k$ intensity of capital, $k=K / L ; K=\mathbf{q A p}$ total capital, $L=\mathbf{q} \mathbf{l}$ labour employed. Hence $k=(1 / r)(y-w)$. Clearly, $k$ is given by $\tan \varphi$ at each rate of profit in Diagram Note 3, showing the wage curve of a given technique:

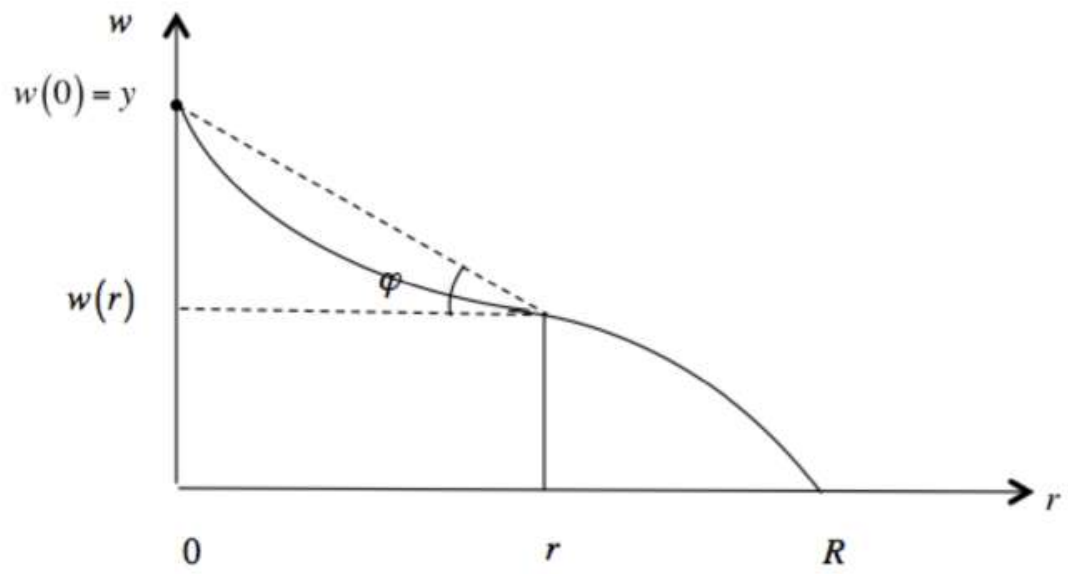

Diagram Note 3: Wage curve of a given technique with capital-intensity $\tan \varphi$ at $r$.

${ }^{4}$ If there are several techniques producing the same goods, each will be characterized by its own wage curve. The profit-maximizing technique will at each $r$ be that of the wage curve on the envelope. Wage curves intersecting on the envelope have generically all methods in all industries in common, except in one: the method is changed only in one industry. The intersection where the method change occurs is called a switch-point. If the wage curves happen to be straight lines as in the case of the wage curves $w^{3}$ and $w^{4}$ in Diagram Note 4 , the capital-intensity does not change along the wage curve, and increasing $r$ means the adoption of wage curves of lower capital intensity, as $r$ rises and $w$ falls at the switch-point $D$ on the envelope. This inverse relationship between the capital-intensity and the rate of profit does not hold between $A$ and $B$ along wage curve $w^{2}$ because of its concave curvature: the intensity of capital rises between $A$ and $B$. This is a non-neoclassical Wicksell-effect. Moreover, the intensity of capital rises at $B$ in the transition from $w^{2}$ back to $w^{1}$ on the envelope (so-called reswitching).

${ }^{5}$ Before hinting at generalisations, Samuelson worked with a two-sector model of a capital good, used in its own reproduction and for the production of a consumption good, which became the numéraire. He supposed that the capital good was, for each technique, specific for the production of the consumption good, that is, the capital good of one technique could not be used in combination with the method to produce the consumption good of another technique.

Wage curves are straight lines, if and only if either the numéraire is an eigenvector of the input-output matrix (Sraffa's standard commodity) or if relative prices are constant for all $r$, hence if they are equal to prices at $r=0$, 


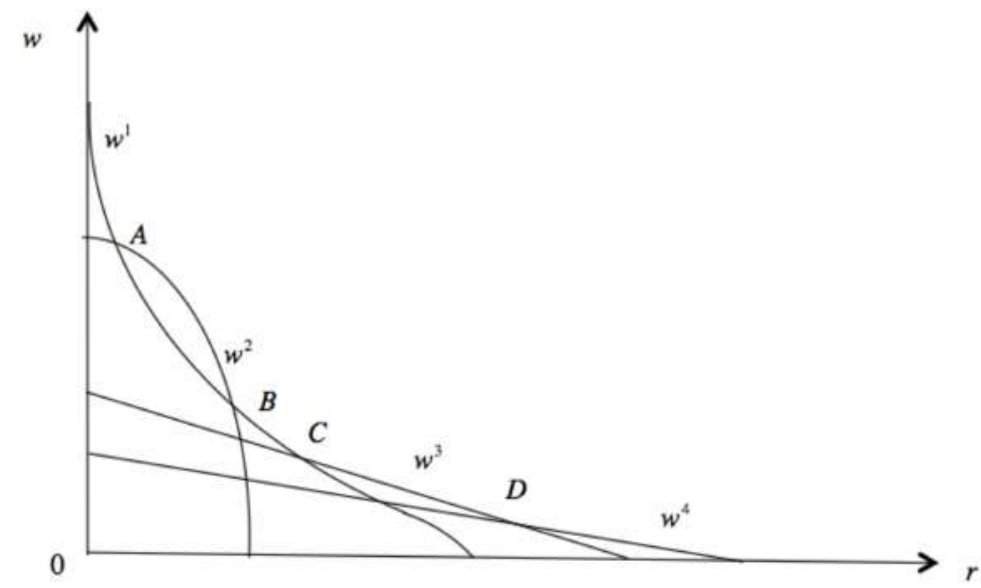

Diagram Note 4: Four wage curves, $w^{1}$ and $w^{2}$ curved, $w^{3}$ and $w^{4}$ linear. Intensity of capital falls with rising $r$ in line with neoclassical theory at $D$. Paradoxical effects due to the curvatures of $w^{1}$ and $w^{2}$.

which means that they are equal to labour values. Samuelson did not make it clear that this was his assumption. It has been pointed out by Salvadori and Steedman (1988) that the intersection of the linear wage curves of two systems on the Samuelson's envelope would be dominated by combinations of the methods taken from the two systems, but Samuelson had excluded such combinations by this assumption of specificity.

${ }^{6}$ Reswitching has been illustrated in note 4 . Reverse capital deepening occurs, if there is a third wage curve dominating the first but not the second switch in what would otherwise, without the third wage curve, be reswitching. One then has an envelope with all switches except one being of the neoclassical type, and the switch where the intensity of capital unexpectedly rises is not marked as a return of a technique that would be visible on the envelope.

${ }^{7}$ If a large number of straight individual wage curves (as above $w^{3}$ and $w^{4}$ in Note 5) of individual techniques are given, such that each is in part on the envelope, the envelope itself constitutes a collective wage curve for the entire spectrum of techniques. It is monotonically falling and convex. Making the transition to a continuum of techniques, one gets a smooth wage curve as envelope $\bar{w}(r)$, with $\bar{w}^{\prime}(r)<0$ and $\bar{w}^{\prime \prime}(r)>0$. The absolute value of the slope of the tangent $-\bar{w}^{\prime}(r)=k$ is the capital-intensity of the technique. If one now defines a per capita function $f(k)=\bar{w}(r)+r k(r)=\bar{w}(r)-r \bar{w}^{\prime}(r)$, one finds

$$
\frac{d f}{d k}=\frac{d f}{d r} / \frac{d k}{d r}=\left(\bar{w}^{\prime}(r)-\bar{w}^{\prime}(r)-r \bar{w}^{\prime \prime}(r)\right) /\left(-\bar{w}^{\prime \prime}(r)\right)=r
$$

and

$$
\frac{d^{2} f}{d k^{2}}=-\frac{1}{\bar{w}^{\prime \prime}(r)}<0 .
$$

Extending to $F(K, L)=L f(k)$, one therefore has constructed a production function with constant returns to scale and with diminishing marginal products. The converse, the derivation of the wage curve from the production function fulfilling the marginal productivity conditions is obvious and yields $-\bar{w}^{\prime}(r)=k>0$ and $\bar{w}^{\prime \prime}(r)>0$. (The argument is also used in the proof of Proposition 4.)

The difficulties discussed in the critique arise, if the individual wage curves are not straight. Suppose the envelope, the collective wage curve $\bar{w}(r)$, is tangent to an individual wage curve $\tilde{w}(r)$ at some $\bar{r}$. According to the construction of the production function, we must have $-\bar{w}^{\prime}(\bar{r})=-\tilde{w}^{\prime}(\bar{r})=k$, but, for the individual wage curve, $k$ is given by $k=(1 / r) /(\tilde{w}(0)-\tilde{w}(r))$, according to Diagram Note 3 . The discrepancy between the two determinations of $k$ is called declination; it is illustrated in Diagram Note 7:

Declination, the discrepancy of $k=\tan \alpha$ and $k=\tan \beta$, could be zero by coincidence, if $\tilde{w}(0)$ happened to coincide with point $P=\bar{r} \tan \alpha$, although $\tilde{w}(r)$ is not a straight line, but if we neglect the possibility of the coincidence (which has mostly been overlooked in the literature), it is clear that straight individual wage curves are not only sufficient, but also necessary for the neoclassical paradigm to hold. Wicksell effects, reswitching and reverse capital deepening are other manifestations of the problem of non-linear wage curves.

${ }^{8}$ See also Note 5 above 


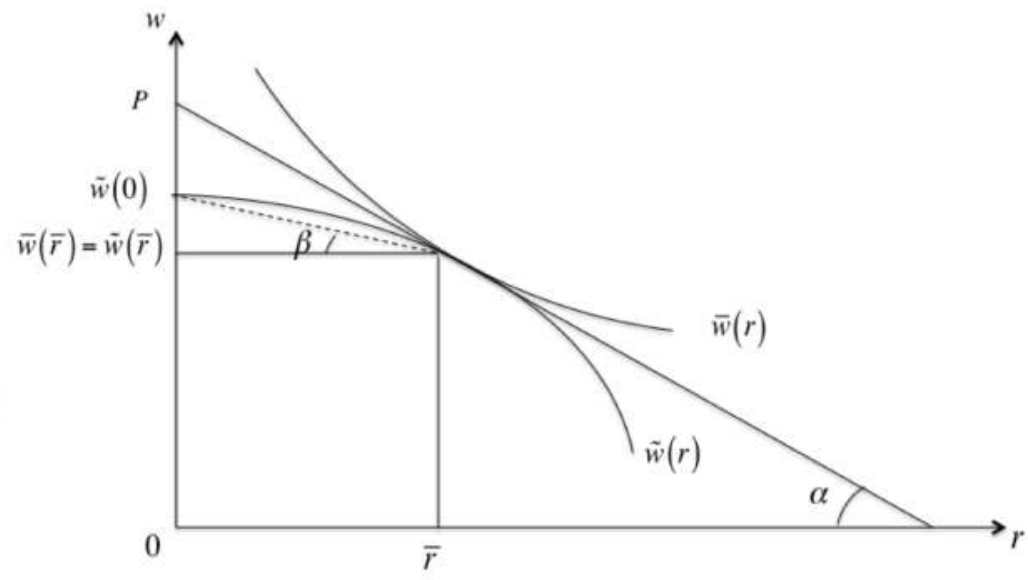

Diagram Note 7: If there is a continuum of techniques and if the wage curve of each technique is a straight line, it is tangent to the collective wage curve $\bar{w}$ as shown in the digram at $\bar{r}$. Output per head then is equal to $P$ and the capital-labour ratio is given by $\tan \alpha$. But if the individual wage curves are not straight, like wage curve $\tilde{w}$ at $\bar{r}$, output per head is given by $\tilde{w}(0)$ and the capital-labour ratio is given by $\tan \beta$. The fact that there is a difference between $P$ and $\tilde{w}(0)$ and between $\tan \alpha$ and $\tan \beta$ is called declination.

${ }^{9}$ On the one hand, one has to admit that methods of production are always adapted to local conditions (institutions, geographical givens). On the other, combinations are the essential result of competition and emerge up to today in the process of globalisation. One of the most eminent and influential present-day Chinese economists writes: "Technological innovation: borrowing is the preferred option." (Lin 2012, p. 13). This refers primarily to the copying and developing of industrial processes of the more advanced countries by those, who catch up. It is typical that the technique to be copied is the dominating technique, the most advanced technique, and not one that would be less efficient and more labour-intensive. The Chinese do not copy techniques that are twenty years old, but the most recent ones, if they can, although they still live in a labour surplus economy.

${ }^{10}$ We recall that Samuelson postulated specificity (Samuelson 1962, p. 196), while Schumpeter postulated that technical change came about through 'new combinations' (Schumpeter 1969 [1934], pp. 12-16).

${ }^{11}$ If $\mathbf{A}$ is diagonalisable, with eigenvalues $\mu_{1}, \ldots, \mu_{n}$, left-hand eigenvectors (rows) $\mathbf{q}_{i} ; \mathbf{q}_{i} \mathbf{A}=\mu_{i} \mathbf{q}_{i}$, and righthand eigenvectors $\mathbf{x}^{i} ; \mathbf{A} \mathbf{x}^{i}=\mu_{i} \mathbf{x}^{i} ; i=1, \ldots, n$; and if we write $\mathbf{l}=\mathbf{x}^{1}+\cdots+\mathbf{x}^{n}$, where, for convenience, the $\mathbf{x}^{i}$ are so normalized that the coefficients in the representation of $\mathbf{l}$ as a linear combination of the $\mathbf{x}^{i}$ are all equal to unity, we have

$$
\mathbf{p}=(\mathbf{I}-(1+r) \mathbf{A})^{-1} \mathbf{l}=w \sum_{i=1}^{n} \frac{\mathbf{x}^{i}}{1-(1+r) \mu_{i}} .
$$

If $\mu_{1}>0$ is the Frobenius eigenvalue with $\mathbf{q}_{1}>0$ and $\mathbf{x}^{1}>0$, we get, with $\mathbf{q}_{1}$ as numéraire vector, because $\mathbf{q}_{1} \mathbf{x}^{j}=0 ; j=2, \ldots, n$;

$$
1=\mathbf{q}_{1} \mathbf{p}=\frac{w \mathbf{q}_{1} \mathbf{x}^{1}}{1-(1+r) \mu_{1}}=w \frac{1+R}{R-r} \mathbf{q}_{1} \mathbf{x}^{1}
$$

with $\mu_{1}=1 /(1+R) ; R$ maximum rate of profit. Hence

$$
w=\frac{R-r}{(1+R) \mathbf{q}_{1} \mathbf{x}^{1}},
$$

which is Sraffa's linear wage curve, except for the difference in normalisation.

${ }^{12}$ Whether the deviations from linearity are strong or weak is a matter of judgement. See Mariolis and Tsoulfidis (2014).

${ }^{13}$ This has been maintained consistently by Anwar Shaikh and his school (Shaikh 2016).

${ }^{14}$ The eigenvalues of large random matrices tend to zero (Goldberg and Neumann 2003), and if we set them to 
zero, the formula for prices of Note 11 becomes

$$
\mathbf{p}=w\left[\frac{1+R}{R-r} \mathbf{x}^{1}+\mathbf{x}^{2}+\cdots+\mathbf{x}^{n}\right] .
$$

Let net output vector $\mathbf{d}$ be written as $\mathbf{d}=\mathbf{q}_{1}+\cdots+\mathbf{q}_{n}$ in analogy to the representation of $\mathbf{l}$ in Note 10 . The wage in terms of $\mathbf{d}$ then is

$$
w=\frac{1}{\mathbf{d} \hat{\mathbf{p}}}=\frac{1}{\frac{1+R}{R-r} \mathbf{q}_{1} \mathbf{x}^{1}+\mathbf{q}_{2} \mathbf{x}^{2}+\cdots+\mathbf{q}_{n} \mathbf{x}^{n}}
$$

The wage curve is linear, if

$$
\mathbf{q}_{2} \mathbf{x}^{2}+\cdots+\mathbf{q}_{n} \mathbf{x}^{n}=\left(\mathbf{d}-\mathbf{q}_{1}\right)\left(\mathbf{l}-\mathbf{x}^{1}\right)=0,
$$

therefore if the deviations $\mathbf{m}=\mathbf{d}-\mathbf{q}_{1}$ of the 'Sraffa vector' $\mathbf{q}_{1}$ from the numéraire vector $\mathbf{d}$ and of the 'Marx vector' $\mathbf{x}^{1}$ from the labour vector $\mathbf{l}, \mathbf{v}=\mathbf{l}-\mathbf{x}^{1}$ are orthogonal, and this will be the case if $\mathbf{m}$ and $\mathbf{v}$ are uncorrelated, for then, $\mathbf{m v}=n \bar{m} \bar{v}$ and $\bar{v}$ can be shown to vanish, if $\mathbf{A}$ is random. An 'approximate' surrogate production function is proposed on the basis of these assumptions, leading to linear wage curves in Schefold (2012).

${ }^{15}$ In between the totally disaggregated general equilibrium model of the Arrow-Debreu type and the aggregate production function models we find the early neoclassical general equilibrium models described by Garegnani (1960) and and Petri (2004), in which distribution between capital and labour is analysed by assuming that a nominal quantity of capital, fixed in terms of the numéraire, is given as the supply. The demand for capital goods follows from the conditions of reproduction in a steady state, and the endowments permitting the maintenance of the steady state are endogenous. It can be shown that the conditions for such an equilibrium to exist and to fulfill appropriate stability conditions are very similar to those needed to prove the possibility of aggregation of a production function (Schefold 2016).

${ }^{16}$ Samuelson's interpretation of the maximum rate of profit as the maximum rate of growth is, of course, formally correct but the comparisons here made refer to stationary systems, as he himself pointed out. We could therefore also contrast the productivity of labour with the productivity of capital, for the maximum rate of profit gives profit divided by capital, when profit absorbs the entire product. (The maximum rate of profit can by the way also be seen as the inverse capital-output ratio, with capital goods valued at the prices pertaining to this maximum rate.)

${ }^{17}$ To test our results empirically by analysing potential combinations chosen from actual input-output tables will be a coming task, which requires preparations. How to calculate the coefficients from monetary data so that they become comparable, how to deal with fixed capital, foreign trade, services and public administration in a theory based on the paradigm of an industrial economy these are questions for which solutions have been proposed by Shaikh (2016), Mariolis and Tsoulfidis (2014) and others. The main difficulty here is that actual wage curves may be quasi-linear, but they are not strictly linear. An empirical investigation would require an adaption of our results to quasi-linear wage curves, appropriately defined.

${ }^{18}$ Strictly speaking, $m$ measures the number of grid lines counted along $F G$ and $F D$ respectively, so that $m$ should more rigorously be defined as the largest natural number smaller than $\sqrt{\gamma s}$.

${ }^{19}$ Note that the square root here imposes itself. For if one lets $m$ grow with $s$, using $m=s^{\alpha}$, it is clear that $\alpha>0$ (otherwise no growth) and $\alpha<1$ (otherwise $m$ overtakes $s$ ). The convergence of

$$
\exp \left[\frac{m(m+1)}{2(s-m)}\right]=\exp \frac{s^{2 \alpha}+s^{\alpha}}{2 s-2 s^{\alpha}}=\exp \frac{1+s^{-\alpha}}{2 s^{1-2 \alpha}-2 s^{-\alpha}}
$$

then requires $\alpha=1 / 2$.

${ }^{20}$ Putting $w^{s}(0)=0$ for simplicity

${ }^{21}$ Anwar Shaikh (1987) has shown that this growth process with constant shares and a rising capital-intensity creates the illusion of a Cobb-Douglas production function.

${ }^{22}$ If the central hypothesis is replaced by the assumptions discussed under 'Second' above, that is, if we have $P\left(R_{\sigma}>R_{\sigma-1}, \ldots, R_{\sigma}>R_{1}\right)=1 / \sigma^{\beta}, \beta>0, \beta \neq 1$ for all $\sigma$, we get, with the same subdivision for the sequence of wage curves as above:

$$
\begin{aligned}
\omega & =\omega^{\prime}+\omega^{\prime \prime}=1+\frac{1}{2^{\beta}}+\frac{1}{3^{\beta}}+\cdots+\frac{1}{s^{\beta}} \cong \frac{s^{1-\beta}-1}{1-\beta}, \\
\omega^{\prime} & =1+\frac{1}{2^{\beta}}+\cdots+\frac{1}{(\vartheta s)^{\beta}} \cong \frac{(\vartheta s)^{1-\beta}-1}{1-\beta}, \\
\omega^{\prime \prime} & =\frac{1}{(\vartheta s)^{\beta}+1}+\cdots+\frac{1}{s^{\beta}} \cong \frac{s^{1-\beta}}{1-\beta}\left(1-\vartheta^{1-\beta}\right) .
\end{aligned}
$$

In this case, $\omega^{\prime \prime}$ tends to infinity, and we cannot prove that the clustering takes place exclusively at the ends, as $s \rightarrow \infty$. We can use Zambelli's (2017) diagram once more to illustrate the possibilities. It shows the envelope of the techniques derived from the input-output tables of 30 countries with 31 sectors, hence $s=30^{31} \approx 6.2 \cdot 10^{45}$ 
and 63 wage curves appear on the envelope, with 62 switch points. If we make the central assumption and apply the formula of the Theorem, we obtain $\frac{2}{3} \ln \left(30^{31}\right) \cong 70$, which is plausible. We can also ask what $\beta$ would have to be, if the formula for $\omega$ above is to hold. It implies a $\beta$ quite close to 1 , justifying the use of the logarithmic approach with its logical stringency.

${ }^{23}$ We have made use of Zambelli (2017) several times, although his own interpretation of his results differs somewhat from ours, as is clear especially from his parallel paper (Zambelli 2018); we briefly clarify our position. Zambelli uses his ingenious algorithm to calculate the envelope of wage curves in both papers; the algorithm is published in Zambelli 2017, pp. 51-55. His critique of neoclassical theory, based on the analysis of the envelopes derived by means of the algorithm, uses two types of analyses, a direct and an indirect one. The direct method, explained in Zambelli (2018, p. 395 and Note 21) calculates the relevant magnitudes such as capital directly from the data. In particular, it computes isoquants, keeping the value of net output constant. The prices are those pertaining to the technique chosen at that rate of profit, but the composition of output is that which minimizes cost of production (equation 24). This implies that iso-value curves can be derived, which can be compared with the isoquants derived from a neoclassical production function. Zambelli finds that they are often not falling. At a switch between different techniques on the envelope, keeping this net output value constant, the value of capital often rises. Zambelli interprets this phenomenon as a critique of neoclassical theory and speaks of capital reversals in this context, but I find this argument problematic. His construction of "iso-value" implies that net output changes between the techniques, insofar as the net output vector changes and only the value in terms of numéraire is preserved. But to trace an isoquant means to keep real net output constant. What is more, the composition of output here follows from the minimization of costs and not, as it should, from the demand for a certain composition of net output. That the minimization of cost should dictate what people consume (or the proportions in which they consume) is neither a neoclassical, nor a classical or Keynesian proposition, nor is it an expression of realism. The consequences of this assumption can therefore not be accepted as a critique of neoclassical theory. Zambelli himself admits that his derivation is formal and does not imply anything about "actual market behaviour" (Zambelli 2018, p. 395), but what, then, is the relevance of the construct? The direct method also is used to calculate wage curves etc., if the vector of real net output is given. It then must lead for mathematical reasons to the same results as the less cumbersome indirect method, to which we now turn.

By contrast with what was said about the iso-value case, we can agree with Zambelli's "indirect" method, based on the analysis of the wage curves, as in this paper (compare Notes 3 and 7 with the corresponding diagrams). It is clear that declination will arise as soon as wage curves are not linear, and this will happen not only if there are non-neoclassical Wicksell effects as in Diagram Note 7, but also, if the curvature corresponds to a neoclassical Wicksell effect (Diagram Note 3).

The capital-labour ratio or capital intensity can be read from the wage curve diagram, using the formula $k=(1 / r)(y-w)$, as we have seen. The isoquants can also be read off the wage curves, if $\mathbf{d}$ is the composition of net output and the numéraire with $\mathbf{d p}=1$. We connect $y=\mathbf{d p} / \mathbf{q} \mathbf{l}=w(0)=1 / L$, where $L$ is labour employed, and $w(r)$ by a straight line $g$. The line $g$ cuts the abscissa at $x$. We then have $k=K / L=y / x$, hence $K=1 / x$ and $L=1 / y$. One can now derive how capital $K$ and labour $L$ change with changes in the rate of profit in the production of a unit output from a wage curve diagram (which the readers will draw for themselves). Labour stays constant along a given wage curve, because the same unit output is produced by means of the same technique. At a switchpoint on the envelope, output per head falls, hence the amount of labour employed rises. Capital changes along a given wage curve as the capital labour ratio; it falls along a wage curve with a neoclassical Wicksell effect and rises in the opposite case. As we may expect and as we continue to assume, the wage curve diagram is disentangled. Therefore capital will fall at switchpoints and labour rise, so that there are capital reversals along wage curves with non-neoclassical Wicksell effects, but no capital reversals at switchpoints. If one now looks at Zambelli's diagram in Zambelli 2017 (2017, p. 41), one finds all these properties confirmed by close inspection. The critique of neoclassical theory rests on the non-neoclassical Wicksell effects. Reswitching and reverse capital deepening (see Notes 4 and 6) are a theoretical possibility, but they are not encountered here.

The most interesting aspect of the diagram, from the point of view of this paper, concerns the counting of the wage curves on the envelope. 63 such curves appear on it. It is the envelope of $30^{31}$ wage curves, since there are 30 countries and 31 sectors (Zambelli speaks of $31^{30}$ wage curves on p. 42, which is a slip). The formula of Kersting's theorem predicts $(2 / 3) \ln \left(30^{31}\right) \approx 70$ wage curves on the envelope; the estimate of the upper bound of the expected number of wage curves on the envelope according to Proposition 1 is $\ln \left(30^{31}\right) \approx 105$.

The data of this diagram refer to the year 2011. Zambelli also considers earlier years in the period 1995-2011, in order to analyse technical progress, which is very interesting, but not of our concern at present. He calculates an intertemporal wage profit frontier, based on 15 years of observation, which by definition dominates the wage profit frontiers of individual years, since in each industry one method taken from 30 countries and 15 years may be chosen, so that there are in total $(30 \cdot 15)^{31}$ wage curves. The resulting intertemporal envelope is composed of 100 wage curves. The formula of Kersting's theorem yields $(2 / 3) \ln \left((30 \cdot 15)^{31}\right) \approx 126$ wage curves. Since there is progress during the time period and since the countries are somewhat unequal, it is not surprising that the theoretical formula predicts a somewhat larger number of wage curves on the envelope than are actually found. 
These results have been obtained, although Kersting's theorem assumes linear wage curves; the approximation of empirical wage curves by linear ones is successful to this extent. Zambelli's diagram (2017, p. 41) also shows that there is a certain flattening of the envelope in its middle, while it is less clear whether the switchpoints are more dense in the corners, as the theorem also predicts. Whether this is due to the fact that wage curves are not linear or that they are (on the envelope) not very numerous or whether yet another influence plays a role in this remains to be investigated.

\section{References}

Aghion, Philippe and Howitt, Peter (1998): Endogenous Growth Theory. Massachusetts Institute of Technology.

Bliss, Christopher J. (1970): Comment on Garegnani. Review of Economic Studies, vol. 17, pp. 437-438.

Garegnani, Pierangelo (1960): Il Capitale nelle Teorie della Distribuzione. Milan: Giuffrè.

Garegnani, Pierangelo (1970): Heterogeneous Capital, the Production Function and the Theory of Distribution, Review of Economic Studies, vol. 37 (3), pp. 407-436.

Garegnani, Pierangelo (1976): On a Change in the Notion of Equilibrium in Recent Work on Value and Distribution, in: Murray Brown, Kazuo Sato, and Paul Zarembka (eds.), Essays on Modern Capital Theory. Amsterdam: North-Holland Publication, pp. 25-45.

Goldberg, G. and Neumann, M. (2003): Distribution of Subdominant Eigenvalues of Matrices with Random Rows. Society for Industrial and Applied Mathematics Journal on Matrix Analysis and Application 24 (3), pp. $747-761$.

Hagemann, Harald (2020): The Cambridge-Cambridge Controversy on the Theory of Capital: 50 Years After. Introduction. European Journal of Economics and Economic Policies, vol. 17 (2), pp. 196-207.

Han, Zonghie (2003): "Paradoxa" in der Kapitaltheorie: eine empirische Untersuchung der Reverse Capital Deepening- und Reswitching-Phänomene anhand der linearen Programmierung im Rahmen der Kapitalkontroverse. Marburg: Metropolis.

Han, Zonghie and Schefold, Bertram (2006): An Empirical Investigation of Paradoxes: Reswitching and Reverse Capital Deepening in Capital Theory. Cambridge Journal of Economics, vol. 30 (5), pp. 737-765.

Harcourt, Geoffrey C. (1972):Some Cambridge Controversies in the Theory of Capital. Cambridge: Cambridge University Press.

Kurz, Heinz; Salvadori, Neri (1999): Theory of Production. A Long-Period Analysis. Cambridge: Cambridge University Press.

Kurz, Heinz, ed. (2000): Critical Essays on Piero Sraffa's Legacy in Economics. Cambridge: Cambridge University Press.

Kurz, Heinz (2020): The Theory of Value and Distribution and the Problem of Capital. European Journal of Economics and Economic Policies, vol. 17 (2), pp. 241-264.

Levhari, David (1965): A Nonsubstitution Theorem and Switching of Techniques. The Quarterly Journal of Economics, vol. 79 (1), pp. 98-105. 
Lin, Justin Yifu (2012): Demystifying the Chinese Economy. Cambridge: Cambridge University Press.

Mainwaring, Lynn and Steedman, Ian (2000): On the Probability of Reswitching and Capital Reversing in a Two-sector Sraffian Model, in: Kurz, H. (ed.) (2000): Critical Essays on Piero Sraffa's Legacy in Economics. Cambridge: Cambridge University Press, pp. 323-354.

Mariolis, Theodore and Tsoulfidis, Lefteris (2014): On Brdy's Conjecture: Theory, Facts and Figures about Instability of the US Economy. Economic Systems Research, 26 (2), pp. 209-223.

Pasinetti, Luigi L. (1966): Changes in the Rate of Profits and Switches of Techniques. The Quarterly Journal of Economics, vol. 80 (4), pp. 503-517.

Petri, Fabio (2004): General Equilibrium, Capital and Macroeconomics: A Key to Recent Controversies in Equilibrium Theory. Cheltenham, UK and Northampton, MA: Edward Elgar Publishing.

Petri, Fabio (2011): On the Likelihood and Relevance of Reswitching and Reverse Capital Deepening, in: Salvadori, N., Gehrke, C. (eds.), Keynes, Sraffa and the Criticism of Neoclassical Theory: Essays in Honour of Heinz Kurz. London and New York: Routledge, pp. 380-418.

Robinson, Joan (1953-1954): The Production Function and the Theory of Capital. Review of Economic Studies, vol. 21, pp. 312-320.

Salvadori, Neri and Steedman, Ian (1988): No Reswitching? No Switching. Cambridge Journal of Economics, 12 (4), pp. 481-486.

Samuelson, Paul (1962): Parable and Realism in Capital Theory: The Surrogate Production Function. Review of Economic Studies, 1962, vol. 29 (3), pp. 193-206.

Samuelson, Paul (1966): A Summing Up. The Quarterly Journal of Economics, vol. 80 (4), pp. 568-583.

Samuelson, Paul (1966 [1961]): Economists and the History of Ideas (Presidential Address, A.E.A. 1961), in: Joseph E. Stiglitz, (ed.), The Collected Scientific Papers of Paul A. Samuelson, Massachusetts Institute of Technology, pp. 1499-1516.

Schefold, Bertram (1976): Different Forms of Technical Progress, The Economic Journal, 86, pp. 806819 .

Schefold, Bertram (2008): Savings, Investment and Capital in a System of General Intertemporal Equilibrium: An Extended Comment on Garegnani with a Note on Parrinello, in: Guglielmo Chiodi and Leonardo Ditta (eds.), Sraffa or an Alternative Economics. Basingstoke: Macmillan, pp. 127-184.

Schefold, Bertram (2013a): Approximate Surrogate Production Functions, Cambridge Journal of Economics, 37 (5), pp. 947-983.

Schefold, Bertram (2013b): Only a Few Techniques Matter! On the Number of Curves on the Wage Frontier, in: Levrero, E., Palumbo, A. and Stirati, A. (eds.), Sraffa and the Reconstruction of Economic Theory: Volume One, Theories of Value and Distribution. Basingstoke, UK: Palgrave Macmillan, pp. 46-69. 
Schefold, Bertram (2016): Marx, the Production Function and the Old Neoclassical Equilibrium: Workable under the Same Assumptions? With an Appendix on the Likelihood of Reswitching and Wicksell Effects. Centro Sraffa Working Papers, No 18, March. Online as Centro Sraffa Working Papers, ISSN $2284-2845$.

Schefold, Bertram (2017): Pluralism in Economics: The Case of the Cambridge School. Proposal for a Transformation of the Critique of Capital, in: Pieter Hennipman Stichting (ed.) Het Gesproken Woord. Pieter Hennipman Diner 2017. Amsterdam, pp. 16-46.

Schefold, Bertram (2018): The Improbability of Reswitching, the Certainty of Wicksell-Effects and the Poverty of Production Functions: The Cambridge Critique of Capital Transformed, in: Luca Fiorito, Scott Scheall and Carlos E.Suprinyak, (eds.). Research in the History of Economic Thought and Methodology, vol. 35B, including a Symposium on New Directions in Sraffa Scholarship. Bingley, UK: Emerald Publishing Limited 2018, pp.171-194.

Schefold, Bertram (2020): What Remains of the Cambridge Critique of Capital Theory, if Reswitching and Reverse Capital Deepening are Empirically Rare and Theoretically Unlikely? European Journal of Economics and Economic Policies, vol. 17 (2), pp. 220-240.

Schumpeter, Joseph Alois (1969 [1934]): The Theory of Economic Development, transl. by Redvers Opie, Reprint. London: Oxford University Press.

Shaikh, Anwar (1987): Humbug Production Function. The New Palgrave. A Dictionary of Economics, vol. 2. London: Macmillan, pp. 690-691.

Shaikh, Anwar (2016): Capitalism: Competition, Conflict, Crises. Oxford: Oxford University Press.

Shaikh, Anwar; Jos Alejandro Coronado; Luiza Nassif-Pires (2020): On the Statistical Regularities of Prices of Production. European Journal of Economics and Economic Policies, vol. 17 (2), pp. 265-275.

Sraffa, Piero (1960): Production of Commodities by Means of Commodities. Cambridge: Cambridge University Press.

Weizsäcker, Carl Christian von (1971): Steady State Capital Theory. (Lecture Notes in Operations Research and Mathematical Systems; 54). Berlin: Springer.

Weizsäcker, Carl Christian von (2020): Böhm-Bawerk and Hicks Modernized. European Journal of Economics and Economic Policies, vol. 17 (2), pp. 208-219.

Zambelli, Stefano (2018): The aggregate production function is NOT neoclassical. Cambridge Journal of Economics, 42, 2018, pp. 383-426.

Zambelli, Stefano, Thomas Fredholm and Ragupathy Venkatachalam (2017): Robust Measurement of National Technological Progress. Structural Change and Economic Dynamics, vol. 42, pp. 38-55. 
AUTHORS:

Götz Kersting, Bertram Schefold

CORRESPONDING AUTHOR:

Bertram Schefold

Fachbereich Wirtschaftswissenschaften, RuW Fach 70

Johann Wolfgang Goethe-Universit• at

Theodor-W.-Adorno-Platz 4,

60323 Frankfurt am Main,

Tel home +4969555371

Tel o_ce +4969798 34759

Fax +496979834544

schefold@wiwi.uni-frankfurt.de

We thank Christian Bidard for helpful advice. The usual caveat applies. 